\title{
A Super-Martingale Property of the Optimal Portfolio Process
}

\author{
Walter Schachermayer*
}

September 16, 2002

\begin{abstract}
We show that, for a utility function $U: \mathbb{R} \rightarrow \mathbb{R}$ having reasonable asymptotic elasticity, the optimal investment process $\widehat{H} \cdot S$ is a super-martingale under each equivalent martingale measure $\mathbf{Q}$, such that $\mathbf{E}\left[V\left(\frac{d \mathbf{Q}}{d \mathbf{P}}\right)\right]<\infty$, where $V$ is the conjugate function of $U$. Similar results for the special case of the exponential utility were recently obtained by Delbaen, Grandits, Rheinländer, Samperi, Schweizer, Stricker as well as Kabanov, Stricker.

This result gives rise to a rather delicate analysis of the "good definition" of "allowed" trading strategies $H$ for a financial market $S$. One offspring of these considerations leads to the subsequent - at first glance paradoxical - example.

There is a financial market consisting of a deterministic bond and two risky financial assets $\left(S_{t}^{1}, S_{t}^{2}\right)_{0 \leq t \leq T}$ such that, for an agent whose preferences are modeled by expected exponential utility at time $T$, it is optimal to constantly hold one unit of asset $S^{1}$. However, if we pass to the market consisting only of the bond and the first risky asset $S^{1}$, and leaving the information structure unchanged, this trading strategy is not optimal any more: in this smaller market it is optimal to invest the initial endowment into the bond.
\end{abstract}

Key words: utility maximization, incomplete markets, duality.

JEL classification: G11, G12, C61

AMS 1991 subject classifications: Primary 90A09, 90A10; secondary 90C26.

*Support by the Austrian Science Foundation (FWF) under the Wittgenstein-Preis program Z36MAT and grant SFB\#010 and by the Austrian National Bank under grant 'Jubiläumsfondprojekt Number 8699' is gratefully acknowledged. Thanks go to P. Grandits and Th. Rheinländer for discussions on the topic of this paper and specially to Ch. Stricker for pointing out an erroneous argument in a previous version of this paper which circulated since December 2000 under the title "How Potential Investments may Change the Optimal Portfolio for the Exponential Utility". The present version has greatly benefited from numerous suggestions and comments by Y. Kabanov, Ch. Stricker, M. Schweizer, as well as by two anonymous referees, for which I also thank.

${ }^{\dagger}$ Department of Financial and Actuarial Mathematics, Vienna University of Technology, Wiedner Hauptstrasse 8-10/105, A-1040 Vienna, Austria, email: wschach@fam.tuwien.ac.at 


\section{Introduction}

This paper continues the study of optimal investment in incomplete financial markets, where we consider a utility function $U: \mathbb{R} \rightarrow \mathbb{R}$ s.t.

$$
U \text { is differentiable, strictly concave, } U^{\prime}(-\infty)=\infty, U^{\prime}(\infty)=0,
$$

and satisfies the condition of reasonable asymptotic elasticity, i.e.,

$$
\limsup _{x \rightarrow \infty} \frac{x U^{\prime}(x)}{U(x)}<1 \quad \text { and } \quad \liminf _{x \rightarrow-\infty} \frac{x U^{\prime}(x)}{U(x)}>1 .
$$

For the significance of the latter condition in the context of utility maximisation we refer to [S 01]. The arch-example of a function $U$ satisfying (1) and (2) is the exponential utility $U(x)=-e^{-\gamma x}$, for $\gamma>0$.

We adopt the same setting as in [S 01] to which we refer for unexplained notation: Fixing the time horizon $T \in] 0, \infty\left[,\left(S_{t}\right)_{0 \leq t \leq T}\right.$ will denote a locally bounded $\mathbb{R}^{d}$-valued semimartingale, modeling the price process of $d$ financial assets. We work in discounted terms, i.e., the bond is assumed to be constant.

We denote by $\mathcal{M}^{e}(S)$ (resp. $\mathcal{M}^{a}(S)$ ) the set of probability measures $\mathbf{Q}$ which are equivalent (resp. absolutely continuous with respect) to $\mathbf{P}$ and such that $S$ is a local Q-martingale. We assume that

$$
\mathcal{M}^{e}(S) \neq \emptyset
$$

A basic problem is to find, for given initial endowment $x \in \mathbb{R}$, a trading strategy $\left(H_{t}\right)_{0 \leq t \leq T}$ such that the expected utility of terminal wealth $x+(H \cdot S)_{T}=x+\int_{0}^{T} H_{t} d S_{t}$ becomes maximal:

$$
\mathbf{E}\left[U\left(x+(H \cdot S)_{T}\right)\right] \longrightarrow \max !
$$

We have been deliberately vague on the set of "allowed" trading strategies $H$ over which we maximize in (4); in fact, the choice of the "good definition" of this class of trading strategies is rather subtle and constitutes the main topic of this paper.

The minimal requirement to impose on an "allowed" trading strategy $H$ is that the stochastic integral $(H \cdot S)_{t}=\left(\int_{0}^{t} H_{u} d S_{u}\right)_{t}$ makes sense. Here the theory of stochastic integration (see, e.g., [P 90], [J 79], [RW 87]) tells us exactly what to impose on $H$ : it has to be a predictable $S$-integrable process.

But, of course, this qualitative requirement is not enough as it does not rule out, e.g., doubling strategies, as was noticed by M. Harrison and S. Pliska ([HP 81]). In order to rule out such strategies, some additional condition is needed.

A strong condition is the subsequent concept of admissible strategies as introduced in [HP 81], modeling the situation of an agent with a finite credit line.

Definition 1 A predictable $S$-integrable process $H$ is an admissible trading strategy if the stochastic integral $(H \cdot S)_{t}=\left(\int_{0}^{t} H_{u} d S_{u}\right)_{t}$ is uniformly bounded from below.

This notion turned out to be very useful for no-arbitrage arguments (compare [HP 81], [DS 94] and [DS 98b]). In the context of utility functions $U$ taking finite values 
only on $\mathbb{R}_{+}$, while being $-\infty$ on $\mathbb{R}_{-}$(typical example: $U(x)=\ln (x)$ ), as analyzed, e.g., in [KLSX 91] and [KrS 99], this concept also proved to be the appropriate one.

In the present setting of utility functions $U$ taking finite values on all of $\mathbb{R}$, we also may and do use this class of trading strategies to give a precise meaning to the maximisation problem (4).

Definition 2 We define the value function u associated to the optimization problem (4) by

$$
u(x)=\sup \left\{\mathbf{E}\left[U\left(x+(H \cdot S)_{T}\right)\right], H \text { admissible }\right\}, \quad x \in \mathbb{R} .
$$

Note that the expectation is well-defined (taking possibly the value $+\infty$ ), and that $u(x)$ is an element of $[U(x), \infty]$.

In the present case of utility functions $U$ taking finite values for all $x \in \mathbb{R}$, the class of admissible trading strategies is too narrow to find the optimizer in (5). In general, we cannot expect to find the optimal solution to (5) such that the random variable $(H \cdot S)_{T}$ is uniformly bounded from below. For example, in the classical Merton problem of optimal investment with respect to exponential utility in the Black-Scholes model, the optimal solution is not bounded from below. Hence we have to look for a somewhat broader class of "allowed" trading strategies.

A possible approach is to impose some integrability condition on the process $H \cdot S$. But under which measure? Should we use the original measure $\mathbf{P}$, or some specific equivalent martingale measure $\mathbf{Q}$, or maybe all equivalent martingale measures? This issue was thoroughly addressed in [DGRSSS 02] and we shall elaborate further on this topic.

For a utility function $U: \mathbb{R} \rightarrow \mathbb{R}$ satisfying (1) we denote by $V: \mathbb{R}_{+} \rightarrow \mathbb{R}$ its conjugate function

$$
V(y)=\sup _{x}(U(x)-x y), \quad y>0 .
$$

For example, for $U(x)=-e^{-\gamma x}$ we have $V(y)=\frac{y}{\gamma}\left(\ln \left(\frac{y}{\gamma}\right)-1\right)$. The dual problem to $(5)$ is given by

$$
v(y)=\inf _{\mathbf{Q} \in \mathcal{M}^{e}(S)} \mathbf{E}\left[V\left(y \frac{d \mathbf{Q}}{d \mathbf{P}}\right)\right], \quad y>0 .
$$

Throughout the paper we shall make the following assumption:

Assumption 1 For each $y>0$, the dual value function $v(y)$ is finite and the minimizer $\widehat{\mathbf{Q}}(y) \in \mathcal{M}^{e}(S)$ for $(7)$, called the minimax martingale measure, exists.

As shown in [BF 02], Assumption 1 is satisfied under rather mild conditions. We remark that it is straightforward to verify that conditions (1), (2), and Assumption 1 imply the assumptions of Theorem 2.2 of [S 01]; hence under the present assumptions we may apply this theorem. This fact will repeatedly be used below.

Specializing to the case of exponential utilty, it follows from the work of Cziszar [C 75] (see also [BF 02] and [S 01, Remark 2.3]) that Assumption 1 is equivalent to the existence of $\mathbf{Q} \in \mathcal{M}^{e}(S)$ with finite relative entropy

$$
H(\mathbf{Q} \mid \mathbf{P})=\mathbf{E}_{\mathbf{P}}\left[\frac{d \mathbf{Q}}{d \mathbf{P}} \ln \left(\frac{d \mathbf{Q}}{d \mathbf{P}}\right)\right]=\mathbf{E}_{\mathbf{Q}}\left[\ln \left(\frac{d \mathbf{Q}}{d \mathbf{P}}\right)\right]<\infty .
$$


In this case the measure $\widehat{\mathbf{Q}}(y)$ does not depend on $y>0$, and minimizes the relative entropy among all absolutely continuous martingale measures, i.e.,

$$
H(\widehat{\mathbf{Q}} \mid \mathbf{P})=\min _{\mathbf{Q} \in \mathcal{M}^{a}(S)} H(\mathbf{Q} \mid \mathbf{P}) .
$$

Following [DGRSSS 02] we shall call $\widehat{\mathbf{Q}}$ the entropy minimizing local martingale measure.

Turning again to general utility functions $\mathrm{U}$, we shall say that $\mathbf{Q} \in \mathcal{M}^{a}(S)$ has finite $V$-expectation if $\mathbf{E}\left[V\left(\frac{d \mathbf{Q}}{d \mathbf{P}}\right)\right]<\infty$. It follows from the assumption of reasonable elasticity (2) that in this case $\mathbf{E}\left[V\left(y \frac{d \mathbf{Q}}{d \mathbf{P}}\right)\right]<\infty$, for each $y>0$ (see [S 01, Corollary 4.2]).

We now can introduce several possible definitions of "allowed" trading strategies.

Definition 3 Under the above assumptions, fix the initial endowment $x \in \mathbb{R}$.

(i) A predictable, S-integrable process $H$ is in $\mathcal{H}_{1}(x)$, if $U\left(x+(H \cdot S)_{T}\right) \in L^{1}(\mathbf{P})$ and $H \cdot S$ is a super-martingale under the minimax martingale measure $\widehat{\mathbf{Q}}(y)$, where $y=u^{\prime}(x)$.

(ii) A predictable, S-integrable process $H$ is in $\mathcal{H}_{2}(x)$, if $U\left(x+(H \cdot S)_{T}\right) \in L^{1}(\mathbf{P})$ and $H \cdot S$ is a super-martingale under each $\mathbf{Q} \in \mathcal{M}^{a}(S)$ with finite $V$-expectation $\mathbf{E}\left[V\left(\frac{d \mathbf{Q}}{d \mathbf{P}}\right)\right]$.

(iii) A predictable, S-integrable process $H$ is in $\mathcal{H}_{3}(x)$, if $U\left(x+(H \cdot S)_{T}\right) \in L^{1}(\mathbf{P})$, $H \cdot S$ is a super-martingale under each $\mathbf{Q} \in \mathcal{M}^{a}(S)$ with finite $V$-expectation $\mathbf{E}\left[V\left(\frac{d \mathbf{Q}}{d \mathbf{P}}\right)\right]$, and there exists a sequence $\left(H^{n}\right)_{n=1}^{\infty}$ of admissible trading strategies such that

$$
\lim _{n \rightarrow \infty} \mathbf{E}\left[U\left(x+(H \cdot S)_{T} \wedge\left(H^{n} \cdot S\right)_{T}\right)\right]=\mathbf{E}\left[U\left(x+(H \cdot S)_{T}\right)\right]
$$

The classes $\mathcal{H}_{1}^{\prime}(x), \mathcal{H}_{2}^{\prime}(x)$ and $\mathcal{H}_{3}^{\prime}(x)$ are defined by replacing in (i), (ii) and (iii) above the term "super-martingale" by the term "martingale".

We remark that in the case of exponential utility the above concepts do not depend on the initial endowment $x$.

The concept $\mathcal{H}_{1}^{\prime}$ was defined - for the case of exponential utility - under the name $\Theta_{1}$ in [DGRSSS 02]; it also plays (implicitly) an important role in the results of [KrS 99] and [S 01] for the case of general utility functions $U$. As was remarked in [DGRSSS 02], it is not very satisfactory to base the definition of the class of allowed strategies on the knowledge of the dual optimizer.

The concept $\mathcal{H}_{2}^{\prime}$ corresponds to the class $\Theta_{2}$ defined in [DGRSSS 02], where - for the exponential utility - it was required that $H \cdot S$ is a $\mathbf{Q}$-martingale, for each $\mathbf{Q}$ with finite entropy. It follows from [DGRSSS 02] and the recent paper [KS 02b] that this latter concept works well for the exponential utility; but we shall see in Proposition 4 below that, for more general utility functions $U: \mathbb{R} \rightarrow \mathbb{R}$, the concept of a supermartingale is more appropriate than that of a martingale. This led us to define the class $\mathcal{H}_{2}$. 
Definition (iii) is in the spirit of [S 01, Definition 1.3]. In addition to the requirements of definition (ii) we also impose an approximability of $H \cdot S$ by a sequence $H^{n} \cdot S$, where each $H^{n}$ is admissible. We remark that this concept is also related to the class $\Theta_{3}$ defined in [DGRSSS 02].

Theorem 1 below asserts that it does not matter which of the classes $\mathcal{H}_{1}, \mathcal{H}_{1}^{\prime}, \mathcal{H}_{2}$ or $\mathcal{H}_{3}$ (and, in the case of exponential utility, also $\mathcal{H}_{2}^{\prime}$ and $\mathcal{H}_{3}^{\prime}$ ) we choose for the utility maximization problem (4). We always end up with the same maximizer $\widehat{H}$, which in addition satisfies the proper duality relations with respect to the minimax martingale measure $\widehat{\mathbf{Q}}(y)$.

For the case of exponential utility the analogous result was proved in [DGRSSS 02] under a mild additional assumption ( $\widehat{\mathbf{Q}}$ was supposed to satisfy a reverse Hölder condition $R_{L \log L}$ ). This assumption was shown to be superfluous in [KS 02a] (compare also [Str01]).

The main result of the present paper is to establish the analogous result for general utility functions $U: \mathbb{R} \rightarrow \mathbb{R}$ satisfying (1) and (2). For expository reasons, we shall also indicate how the proof specializes to the case of exponential utility as the present arguments are different from those in [DGRSSS 02] and [KS 02b]; we then show how they may be extended to general utility functions. The proof of Theorem 1 relies essentially on the ideas of concatenation and dynamic programming. It turns out that some explicit calculations in the case of exponential utility are replaced by more conceptual arguments in the general setting (thus avoiding some calculations).

Having established the equivalence of the concepts $\mathcal{H}_{1}, \mathcal{H}_{1}^{\prime}, \mathcal{H}_{2}$ and $\mathcal{H}_{3}$ with respect to the utility maximization problem (4) it will be natural to ask whether other (weaker) requirements of "allowed" trading strategies also yield the same conclusion. For example, consider the class of predictable $S$-integrable processes such that $H \cdot S$ is a martingale under some $\mathbf{Q} \in \mathcal{M}^{e}(S)$. This class is closely related to the "workable contingent claims" as introduced in [DS 97]. One also might try variations of this requirement by imposing that $H \cdot S$ is a martingale — or a super-martingale — under some $\mathbf{Q} \in \mathcal{M}^{e}(S)$ with finite $V$-expectation.

Proposition 2, which presents the example described in the abstract, shows in a rather striking way that such hopes are in vain. These concepts do not allow for a good duality theory and lead to paradoxical results from an economic point of view. Two similar examples (Propositions 3 and 4) also show the sharpness of the assertions of Theorem 1.

\section{The Main Result}

Theorem 1 Let $S=\left(S_{t}\right)_{0 \leq t \leq T}$ be a locally bounded $\mathbb{R}^{d}$-valued semimartingale and $U: \mathbb{R} \rightarrow \mathbb{R}$ a utility function satisfying (1), (2), (3) and assumption 1. For $x \in \mathbb{R}$, consider the optimization problem

$$
u_{i}(x)=\sup _{H \in \mathcal{H}_{i}(x)} \mathbf{E}\left[U\left(x+(H \cdot S)_{T}\right)\right] .
$$


For $i=1,2,3$ the optimal solution $\widehat{H}_{i} \in \mathcal{H}_{i}(x)$ exists, is unique (in the sense that the process $\left(\left(\widehat{H}_{i} \cdot S\right)_{t}\right)_{0 \leq t \leq T}$ is unique up to the indistinguishability), coincides for all three cases and therefore may be denoted by $\widehat{H}$. In addition, $\widehat{H}$ is also the unique optimizer in the class $\mathcal{H}_{1}^{\prime}(x)$.

The value function $u(x)$ defined in (5) equals $u_{i}(x)$, for $i=1,2,3$. Letting $y=$ $u^{\prime}(x)$, we have the following duality relation between $\widehat{H}(x)$ and the dual minimizer $\widehat{\mathbf{Q}}(y)$ :

$$
x+(\widehat{H} \cdot S)_{T}=-V^{\prime}\left(y \frac{d \widehat{\mathbf{Q}}(y)}{d \mathbf{P}}\right) \quad \text { and } \quad y \frac{d \widehat{\mathbf{Q}}(y)}{d \mathbf{P}}=U^{\prime}\left(x+(\widehat{H} \cdot S)_{T}\right) .
$$

In the case of the exponential utility function $U(x)=-e^{-\gamma x}, \widehat{H}$ does not depend on $x$; it is also the unique minimizer in the classes $\mathcal{H}_{2}^{\prime}$ and $\mathcal{H}_{3}^{\prime}$; relation (12) specializes to

$$
(\widehat{H} \cdot S)_{T}=-\frac{1}{\gamma} \ln \left(\frac{y}{\gamma} \frac{d \widehat{\mathbf{Q}}}{d \mathbf{P}}\right) \quad \text { and } \quad \frac{d \widehat{\mathbf{Q}}}{d \mathbf{P}}=\frac{\gamma}{y} e^{-\gamma(\widehat{H} \cdot S)_{T}} .
$$

where $y=u^{\prime}(0)$.

The theorem essentially relies on Proposition 1 below which, for the case of exponential utility, was proved by Kabanov and Stricker [KS 02b]. Admitting Proposition 1 for the moment, the argument for Theorem 1 goes as follows.

Proof of Theorem 1 As remarked after Assumption 1 above, the present assumptions imply those of [S 01, Theorem 2.2]. In particular, we know that, for $x \in \mathbb{R}$ and $y>0$, satisfying $u^{\prime}(x)=y$, the process

$$
\widehat{X}_{t}(x)=\mathbf{E}_{\widehat{\mathbf{Q}}(y)}\left[-V^{\prime}\left(y \frac{d \widehat{\mathbf{Q}}(y)}{d \mathbf{P}}\right) \mid \mathcal{F}_{t}\right], \quad 0 \leq t \leq T,
$$

well-defines a $\widehat{\mathbf{Q}}(y)$-martingale, which is of the form $\widehat{X}(x)=x+\widehat{H}(x) \cdot S$, for some $S$-integrable predictable process $\widehat{H}(x)$ and

$$
u(x)=\mathbf{E}\left[U\left(x+(\widehat{H}(x) \cdot S)_{T}\right)\right] .
$$

Proposition 1 asserts that $\widehat{X}_{t}(x)$ is a super-martingale under each $Q \in \mathcal{M}^{a}(S)$ with finite $V$-expectation. Admitting this result, we shall show that $\widehat{H}(x)$ is the unique optimizer in the classes $\mathcal{H}_{1}(x), \mathcal{H}_{1}^{\prime}(x), \mathcal{H}_{2}(x)$ and $\mathcal{H}_{3}(x)$.

The fact hat $\widehat{H}(x)$ belongs to each of these classes is obvious for $\mathcal{H}_{1}(x), \mathcal{H}_{1}^{\prime}(x)$ and $\mathcal{H}_{2}(x)$; as regards $\mathcal{H}_{3}(x)$, it follows from [S 01, Theorem 2.2] that $\widehat{H}(x)$ may be approximated by a sequence of admissible trading strategies in the sense of (10).

To show that $\widehat{H}(x)$ is the unique optimizer in each of these classes, we only have to show this for $\mathcal{H}_{1}(x)$, as this is the largest class.

We shall show that, in fact, the $\widehat{\mathbf{Q}}(y)$-martingale $\widehat{X}(x)=x+\widehat{H}(x) \cdot S$ is optimal among the class of all $\widehat{\mathbf{Q}}(y)$-super-martingales $\left(X_{t}\right)_{0 \leq t \leq T}$ starting at $X_{0}=x$. Indeed, let $X$ be a $\widehat{\mathbf{Q}}(y)$-super-martingale and use the duality relation (see [S 01, Theorem $2.2(\mathrm{i})])$

$$
u(x)=\mathbf{E}\left[U\left(\widehat{X}_{T}(x)\right)\right]=\mathbf{E}\left[V\left(y \frac{d \widehat{\mathbf{Q}}(y)}{d \mathbf{P}}\right)\right]+x y=v(y)+x y .
$$


Applying the inequality

$$
\begin{aligned}
U\left(X_{T}(\omega)\right) & =\inf _{\eta>0}\left\{V(\eta)+X_{T}(\omega) \eta\right\} \\
& \leq V\left(y \frac{d \widehat{\mathbf{Q}}(y)}{d \mathbf{P}}(\omega)\right)+X_{T}(\omega) y \frac{d \widehat{\mathbf{Q}}(y)}{d \mathbf{P}}(\omega),
\end{aligned}
$$

pointwise for $\omega \in \Omega$, we get from the $\widehat{\mathbf{Q}}(y)$-super-martingale property of $X$ that

$$
\begin{aligned}
\mathbf{E}\left[U\left(X_{T}\right)\right] & \leq \mathbf{E}\left[V\left(y \frac{d \widehat{\mathbf{Q}}(y)}{d \mathbf{P}}\right)\right]+y \mathbf{E}_{\widehat{\mathbf{Q}}(y)}\left[X_{T}\right] \\
& \leq \mathbf{E}\left[V\left(y \frac{d \widehat{\mathbf{Q}}(y)}{d \mathbf{P}}\right)\right]+x y=\mathbf{E}\left[U\left(\widehat{X}_{T}(x)\right)\right]
\end{aligned}
$$

where the above estimate shows in particular that $\mathbf{E}\left[U\left(X_{T}\right)_{+}\right]<\infty$, so that $\mathbf{E}\left[U\left(X_{T}\right)\right]$ is well-defined. This readily shows that $\widehat{H}$ is the unique optimizer in the class $\mathcal{H}_{1}(x)$. Hence, for $i=1,2,3$, the value functions $u_{i}(x)$ coincide with the value function $u(x)$ as defined in (5).

As regards the fact that $\widehat{H}(x)$ also is in $\mathcal{H}_{2}^{\prime}(x)$ and $\mathcal{H}_{3}^{\prime}(x)$ for the case of exponential utility, we refer to [DGRSSS 02] and [KS 02b].

Proposition 1 Under the assumptions of Theorem 1, the process $\widehat{X}(x)=x+\widehat{H}(x) \cdot S$, defined by (14), is a super-martingale under $\mathbf{Q}$ for each $\mathbf{Q} \in \mathcal{M}^{a}(S)$ with finite $V$ expectation $\mathbf{E}_{\mathbf{P}}\left[V\left(\frac{d \mathbf{Q}}{d \mathbf{P}}\right)\right]$.

To prepare the proof of Proposition 1 we start with some auxiliary results. The subsequent lemma gives a general characterisation of local martingales $X$ (or, more generally, stochastic integrals of local martingales), which are super-martingales. On the basis of a preliminary version of the present paper [S 02], Kabanov and Stricker as well as E. Strasser have further elaborated on this topic ([KS 02b], [St 02]).

Lemma 1 Let $S=\left(S_{t}\right)_{0 \leq t \leq T}$ be a local martingale on $\left(\Omega, \mathcal{F},\left(\mathcal{F}_{t}\right)_{0 \leq t \leq T}, \mathbf{Q}\right)$, $H$ an $S$-integrable predictable process and $X=H \cdot S$.

If, for every sequence $\left(\tau_{n}\right)_{n=1}^{\infty}$ of $[0, T] \cup\{\infty\}$-valued stopping times increasing to $+\infty$, we have

$$
\limsup _{n \rightarrow \infty} \mathbf{E}_{\mathbf{Q}}\left[X_{\tau_{n}} \mathbf{1}_{\left\{\tau_{n}<\infty, X_{\tau_{n}} \leq 0\right\}}\right]=0,
$$

then $X$ is a local martingale and a super-martingale under $\mathbf{Q}$.

The proof of the lemma relies on the subsequent sublemma which is a straight forward consequence of a result of Ansel-Stricker [AS 94, Proposition 3.3] (compare also $[\mathrm{KS} \mathrm{02b}])$.

Sublemma 1 Let $S=\left(S_{t}\right)_{0 \leq t \leq T}$ be a local martingale and suppose that, for $X=H \cdot S$, there is an integrable random variable $\vartheta \geq 0$ such that $X_{t} \geq-\vartheta$, a.s., for all $0 \leq t \leq T$.

Then $X$ is a local martingale and a super-martingale. 
Proof of Lemma 1 Define the sequence of stopping times by $\sigma_{0}=0, n \geq 1$, and

$$
\sigma_{n}=\inf \left\{t: X_{t} \leq-n\right\}
$$

which increases almost surely to infinity.

We assume that (19) holds true and define the random variables

$$
\vartheta_{n}=\max \left(\left(X_{\sigma_{n}}\right)_{-} \mathbf{1}_{\left\{\sigma_{n}<\infty\right\}}, n\right) .
$$

It follows from (19) that, for $n$ sufficiently large, we have $\mathbf{E}_{\mathbf{Q}}\left[\vartheta_{n}\right]<\infty$.

Note that the stopped process $X^{\sigma_{n}}$ is bounded from below almost surely by $-\vartheta_{n}$; indeed, on $\left\{\sigma_{n}=\infty\right\}$ we have $\inf _{0 \leq t \leq T} X_{t}^{\sigma_{n}} \geq-n$, while on $\left\{\sigma_{n}<\infty\right\}$ we have $\inf _{0 \leq t \leq T} X_{t}^{\sigma_{n}}=X_{\sigma_{n}} \mathbf{1}_{\left\{\sigma_{n}<\infty\right\}} \geq-\left(X_{\sigma_{n}}\right)_{-} \mathbf{1}_{\left\{\sigma_{n}<\infty\right\}}$. Sublemma 1 therefore implies that each $X^{\sigma_{n}}$ is a super-martingale and that $X$ is a local martingale under $\mathbf{Q}$. have

We now show that (19) implies that, for every $[0, T]$-valued stopping time $\sigma$, we

$$
E_{\mathbf{Q}}\left[\left|X_{\sigma}\right|\right]<\infty \text {. }
$$

Indeed, otherwise $\lim _{n \rightarrow \infty} \mathbf{E}\left[\left|X_{\sigma \wedge \sigma_{n}}\right|\right]=\infty$ and, therefore, using the supermartingale property of $X^{\sigma_{n}}$,

$$
\lim _{n \rightarrow \infty} \mathbf{E}\left[\left(X_{\sigma \wedge \sigma_{n}}\right)_{-}\right]=\infty .
$$

Letting $A_{n}=\left\{\sigma_{n-1}<\sigma \leq \sigma_{n}\right\}$, we obtain a partition $\left(A_{n}\right)_{n=1}^{\infty}$ of $\Omega$. For the sequence of stopping times $\kappa_{n}=\sigma \mathbf{1}_{A_{n}}+\infty \mathbf{1}_{\Omega \backslash A_{n}}$ we obtain from (23) that

$$
\sum_{n=1}^{\infty} \mathbf{E}_{\mathbf{Q}}\left[\left(X_{\sigma}\right)_{-} \mathbf{1}_{A_{n}}\right]=-\sum_{n=1}^{\infty} \mathbf{E}_{\mathbf{Q}}\left[X_{\kappa_{n}} \mathbf{1}_{\left\{\kappa_{n}<\infty, X_{\kappa_{n}} \leq 0\right\}}\right]=\infty .
$$

By letting $\tau_{k}=\sum_{n=n_{k}+1}^{n_{k+1}} \sigma \mathbf{1}_{A_{n}}+\infty \mathbf{1}_{\Omega \backslash \bigcup_{n=n_{k}+1}^{n_{k+1} A_{n}}}$ for a sequence $\left(n_{k}\right)_{k=1}^{\infty}$ increasing sufficiently fast to infinity, we arrive at a contradiction to (19), which proves (22).

We are ready to show the super-martingale property of $X$. It suffices to fix stopping times $0 \leq \rho \leq \sigma \leq T$ such that $\rho \leq \sigma_{n_{0}}$, for some $n_{0} \in \mathbb{N}$, and to show that

$$
\mathbf{E}_{\mathbf{Q}}\left[X_{\sigma}-X_{\rho}\right] \leq 0 \text {. }
$$

We deduce from the super-martingale property of $X^{\sigma_{n}}$ that, for $n \geq n_{0}$,

$$
\mathbf{E}_{\mathbf{Q}}\left[X_{\sigma \wedge \sigma_{n}}-X_{\rho}\right] \leq 0 \text {. }
$$

Using (22), we may deduce (25) from (26) and assumption (19) applied to the sequence of stopping times $\rho_{n}=\left(\sigma \wedge \sigma_{n}\right) \mathbf{1}_{\left\{\sigma_{n} \leq \sigma\right\}}+\infty \mathbf{1}_{\left\{\sigma_{n}>\sigma\right\}}$ :

$$
\begin{aligned}
\mathbf{E}_{\mathbf{Q}}\left[X_{\sigma}\right] & =\lim _{n \rightarrow \infty} \mathbf{E}_{\mathbf{Q}}\left[X_{\sigma} \mathbf{1}_{\left\{\sigma_{n}>\sigma\right\}}\right]=\lim _{n \rightarrow \infty} \mathbf{E}_{\mathbf{Q}}\left[X_{\sigma \wedge \sigma_{n}} \mathbf{1}_{\left\{\sigma_{n}>\sigma\right\}}\right] \\
& =\lim _{n \rightarrow \infty}\left(\mathbf{E}_{\mathbf{Q}}\left[X_{\sigma \wedge \sigma_{n}}\right]-\mathbf{E}_{\mathbf{Q}}\left[X_{\sigma \wedge \sigma_{n}} \mathbf{1}_{\left\{\sigma_{n} \leq \sigma\right\}}\right]\right) \\
& \leq \lim _{n \rightarrow \infty}\left(\mathbf{E}_{\mathbf{Q}}\left[X_{\sigma \wedge \sigma_{n}}\right]-\mathbf{E}_{\mathbf{Q}}\left[X_{\left.\left.\rho_{n} \mathbf{1}_{\left\{\rho_{n}<\infty, X_{\rho_{n}} \leq 0\right\}}\right]\right)}\right]\right. \\
& \leq \mathbf{E}_{\mathbf{Q}}\left[X_{\rho}\right] .
\end{aligned}
$$


Remark 1 The sufficient condition (19) also is necessary for a process $X=\left(X_{t}\right)_{0 \leq t \leq T}$ to be a super-martingale: indeed, if $X=\left(X_{t}\right)_{0 \leq t \leq T}$ is a super-martingale and the sequence of $[0, T] \cup\{\infty\}$-valued stopping times $\left(\tau_{n}\right)_{n=1}^{\infty}$ increases to $\infty$, the sequence of random variables $\left(X_{\tau_{n}} \mathbf{1}_{\left\{\tau_{n}<\infty, X_{\tau_{n}} \leq 0\right\}}\right)_{n=1}^{\infty}$ is uniformly integrable, which implies (19).

We have formulated Lemma 1 for processes indexed by the closed time interval $I=[0, T]$. As pointed out by $C$. Stricker, this result also extends to the case of the open time interval $I=[0, \infty[$; we then have to require in condition (19) that the sequence $\left(\tau_{n}\right)_{n=1}^{\infty}$ increases stationarily to $\infty$. Note that in the present case of $I=[0, T] a$ sequence $\left(\tau_{n}\right)_{n=1}^{\infty}$ of $[0, T] \cup\{\infty\}$-valued stopping times, increasing to $\infty$, automatically does so in a stationary way.

We now aboard the proof of Proposition 1 for the special case of the exponential utility $U(x)=-e^{-\gamma x}$ which will rely on the subsequent Lemma 2 pertaining to the well-known technique of "concatenation" (compare [KS 02a, Proposition 4.1]).

Lemma 2 Under the assumptions of Theorem 1, let $\mathbf{Q} \in \mathcal{M}^{a}(S)$ with $H(\mathbf{Q} \mid \mathbf{P})<\infty$ and $\tau$ a $[0, T] \cup\{\infty\}$-valued stopping time. Denote by $\left(Z_{t}\right)_{0 \leq t \leq T}$ and $\left(\widehat{Z}_{t}\right)_{0 \leq t \leq T}$ the density processes corresponding to $\mathbf{Q}$ and $\widehat{\mathbf{Q}}$ respectively, and define the probability measure $\mathbf{Q}^{\tau}$ by the following "concatenation operation":

$$
\frac{d \mathbf{Q}^{\tau}}{d \mathbf{P}}=\left\{\begin{array}{ccc}
Z_{T} & \text { if } & \tau=\infty \\
Z_{\tau} \frac{\widehat{Z}_{T}}{\widehat{Z}_{\tau}} & \text { if } & \tau<\infty
\end{array}\right.
$$

Then $\mathbf{Q}^{\tau} \in \mathcal{M}^{a}(S)$ and $H\left(\mathbf{Q}^{\tau} \mid \mathbf{P}\right) \leq H(\mathbf{Q} \mid \mathbf{P})$.

Proof Note that the random variable $Y=\frac{\widehat{Z}_{T}}{\widehat{Z}_{\tau}} \mathbf{1}_{\{\tau<\infty\}}+\mathbf{1}_{\{\tau=\infty\}}$ solves the conditional minimization problem

$$
\mathbf{E}_{\mathbf{P}}\left[Y \ln (Y) \mid \mathcal{F}_{\tau}\right] \longrightarrow \min ! \text { a.s. on }\{\tau<\infty\}
$$

among all nonnegative random variables $Y$ verifying $\mathbf{E}_{\mathbf{P}}\left[Y \mid \mathcal{F}_{\tau}\right]=1$ a.s., and such that the process $\tau S:=S_{t}-S_{t \wedge \tau}$ "starting at $\tau$ " is a local martingale with respect to the measure $\mathbf{R}$ defined by $\frac{d \mathbf{R}}{d \mathbf{P}}=Y$.

Indeed, suppose there is such a function $Y$ and an $\mathcal{F}_{\tau}$-measurable subset $A \subseteq\{\tau<$ $\infty\}, \mathbf{P}[A]>0$, such that

$$
\mathbf{E}_{\mathbf{P}}\left[\frac{\widehat{Z}_{T}}{\widehat{Z}_{\tau}} \ln \left(\frac{\widehat{Z}_{T}}{\widehat{Z}_{\tau}}\right) \mid \mathcal{F}_{\tau}\right]>\mathbf{E}_{\mathbf{P}}\left[Y \ln (Y) \mid \mathcal{F}_{\tau}\right] \quad \text { a.s. on } \quad A \text {. }
$$

Then the probability measure $\widetilde{\mathbf{Q}}$ defined by

$$
\frac{d \widetilde{\mathbf{Q}}}{d \mathbf{P}}=\widetilde{Z}_{T}=\left\{\begin{array}{ccc}
\widehat{Z}_{T} & \text { on } & \Omega \backslash A \\
\widehat{Z}_{\tau} Y & \text { on } & A
\end{array}\right.
$$


would be an element of $\mathcal{M}^{a}(S)$ with smaller entropy than $\widehat{\mathbf{Q}}$ :

$$
\begin{aligned}
& H(\widehat{\mathbf{Q}} \mid \mathbf{P})-H(\widetilde{\mathbf{Q}} \mid \mathbf{P})=\mathbf{E}_{\mathbf{P}}\left[\widehat{Z}_{T} \ln \left(\widehat{Z}_{T}\right)-\widetilde{Z}_{T} \ln \left(\widetilde{Z}_{T}\right)\right] \\
& \quad=\mathbf{E}_{\mathbf{P}}\left[\left(\widehat{Z}_{\tau} \frac{\widehat{Z}_{T}}{\widehat{Z}_{\tau}} \ln \left(\widehat{Z}_{\tau} \widehat{Z}_{T}\right)-\widehat{Z}_{\tau} Y \ln \left(\widehat{Z}_{\tau} Y\right)\right) \mathbf{1}_{A}\right] \\
& \quad=\mathbf{E}_{\mathbf{P}}\left[\widehat{Z}_{\tau} \mathbf{1}_{A} \mathbf{E}_{\mathbf{P}}\left[\frac{\widehat{Z}_{T}}{\widehat{Z}_{\tau}}\left(\ln \left(\widehat{Z}_{\tau}\right)+\ln \left(\frac{\widehat{Z}_{T}}{\widehat{Z}_{\tau}}\right)\right)-Y\left(\ln \left(\widehat{Z}_{\tau}\right)+\ln (Y)\right) \mid \mathcal{F}_{\tau}\right]\right] \\
& \quad=\mathbf{E}_{\mathbf{P}}\left[\widehat{Z}_{\tau} \mathbf{1}_{A} \mathbf{E}_{\mathbf{P}}\left[\frac{\widehat{Z}_{T}}{\widehat{Z}_{\tau}} \ln \left(\frac{\widehat{Z}_{T}}{\widehat{Z}_{\tau}}\right)-Y \ln (Y) \mid \mathcal{F}_{\tau}\right]\right]>0 .
\end{aligned}
$$

This contradiction to the minimality of $\widehat{\mathbf{Q}}$ shows $(29)$.

By the same argument we conclude that $\mathbf{Q}^{\tau}$ is the element of $\mathcal{M}^{a}(S)$ with minimal entropy such that $\left.\mathbf{Q}^{\tau}\right|_{\mathcal{F}_{\tau}}=\left.\mathbf{Q}\right|_{\mathcal{F}_{\tau}}$, which implies the assertion of the lemma.

\section{Proof of Proposition 1 for the case of exponential utility}

Assume that there is $\mathbf{Q} \in \mathcal{M}^{a}(S), H(\mathbf{Q} \mid \mathbf{P})<\infty$, such that $\widehat{X}=x+\widehat{H}$ fails to be a super-martingale under $\mathbf{Q}$. Without loss of generality we may assume that $x=0$ and that $\mathbf{Q} \in \mathcal{M}^{e}(S)$ (consider $\left.\frac{\mathbf{Q}+\widehat{\mathbf{Q}}}{2}\right)$.

From Lemma 1 we deduce that, if $\widehat{X}$ fails to be a Q-super-martingale, there exists a sequence $\left(\tau_{n}\right)_{n=1}^{\infty}$ of stopping times increasing to infinity such that

$$
\liminf _{n \rightarrow \infty} \mathbf{E}_{\mathbf{Q}}\left[-\widehat{X}_{\tau_{n}} \mathbf{1}_{\left\{\tau_{n}<\infty, \widehat{X}_{\tau_{n}} \leq 0\right\}}\right]>0 \text {. }
$$

Of course, we may assume that $\widehat{X}_{\tau_{n}} \leq 0$ on $\left\{\tau_{n}<\infty\right\}$ so that we may replace $\left\{\tau_{n}<\infty, \widehat{X}_{\tau_{n}} \leq 0\right\}$ simply by $\left\{\tau_{n}<\infty\right\}$ in the above formula.

We know that $\widehat{X}$ is a uniformly integrable martingale under $\widehat{\mathbf{Q}}$ and therefore

$$
\lim _{n \rightarrow \infty} \mathbf{E}_{\widehat{\mathbf{Q}}}\left[\widehat{X}_{\tau_{n}} \mathbf{1}_{\left\{\tau_{n}<\infty\right\}}\right]=0
$$

It follows from (33) and (34) that we have

$$
\liminf _{n \rightarrow \infty} \mathbf{E}_{\mathbf{P}}\left[-\widehat{X}_{\tau_{n}} Z_{\tau_{n}} \mathbf{1}_{\left\{\tau_{n}<\infty, Z_{\tau_{n}} \geq \widehat{Z}_{\tau_{n}}\right\}}\right]>0 .
$$

Now apply Lemma 2 to the probability measures $\mathbf{Q}^{n} \in \mathcal{M}^{e}(S)$

$$
\frac{d \mathbf{Q}^{n}}{d \mathbf{P}}=\left\{\begin{array}{ccc}
Z_{\tau_{n}} \frac{\widehat{Z}_{T}}{\widehat{Z}_{\tau_{n}}} & \text { for } & \tau_{n}<\infty \\
Z_{T} & \text { for } & \tau_{n}=\infty
\end{array}\right.
$$

We shall show that

$$
\liminf _{n \rightarrow \infty} H\left(\mathbf{Q}^{n} \mid \mathbf{P}\right)>H(\mathbf{Q} \mid \mathbf{P})
$$


a contradiction to the assertion of Lemma 2 which will finish the proof.

$$
\begin{aligned}
& \liminf _{n \rightarrow \infty}\left(H\left(\mathbf{Q}^{n} \mid \mathbf{P}\right)-H(\mathbf{Q} \mid \mathbf{P})\right) \\
&=\liminf _{n \rightarrow \infty} \mathbf{E}\left[\left(Z_{\tau_{n}} \frac{\widehat{Z}_{T}}{\widehat{Z}_{\tau_{n}}} \ln \left(Z_{\tau_{n}} \frac{\widehat{Z}_{T}}{\widehat{Z}_{\tau_{n}}}\right)-Z_{T} \ln \left(Z_{T}\right)\right) \mathbf{1}_{\left\{\tau_{n}<\infty\right\}}\right] \\
& \geq \liminf _{n \rightarrow \infty}\left(\mathbf{E}\left[Z_{\tau_{n}} \frac{\widehat{Z}_{T}}{\widehat{Z}_{\tau_{n}}} \ln \left(Z_{\tau_{n}} \frac{\widehat{Z}_{T}}{\widehat{Z}_{\tau_{n}}}\right) \mathbf{1}_{\left\{\tau_{n}<\infty, Z_{\tau_{n}} \geq \widehat{Z}_{\left.\tau_{n}\right\}}\right]}\right]+\right. \\
&\left.\quad \quad\left(-\frac{1}{e}\right) \mathbf{P}\left[\tau_{n}<\infty, Z_{\tau_{n}}<\widehat{Z}_{\tau_{n}}\right]-\mathbf{E}\left[Z_{T} \ln \left(Z_{T}\right) \mathbf{1}_{\left\{\tau_{n}<\infty\right\}}\right]\right) \\
&=\liminf _{n \rightarrow \infty} \mathbf{E}\left[Z_{\tau_{n}} \mathbf{1}_{\left\{\tau_{n}<\infty, Z_{\left.\tau_{n} \geq \widehat{Z}_{\tau_{n}}\right\}} \mathbf{E}\left[\frac{\widehat{Z}_{T}}{\widehat{Z}_{\tau_{n}}} \ln \left(\widehat{Z}_{T}\right) \mid \mathcal{F}_{\tau_{n}}\right]\right]}=\liminf _{n \rightarrow \infty} \mathbf{E}\left[Z_{\tau_{n}} \mathbf{1}_{\left\{\tau_{n}<\infty, Z_{\tau_{n}} \geq \widehat{Z}_{\tau_{n}}\right\}} \mathbf{E}\left[\frac{\widehat{Z}_{T}}{\widehat{Z}_{\tau_{n}}}\left(-\gamma \widehat{X}_{T}-\ln \left(\frac{y}{\gamma}\right)\right) \mid \mathcal{F}_{\tau_{n}}\right]\right]\right. \\
&= \liminf _{n \rightarrow \infty} \mathbf{E}\left[Z_{\tau_{n}} \mathbf{1}_{\left\{\tau_{n}<\infty, Z_{\tau_{n}} \geq \widehat{Z}_{\tau_{n}}\right\}}\left(-\gamma \widehat{X}_{\tau_{n}}-\ln \left(\frac{y}{\gamma}\right)\right)\right]>0,
\end{aligned}
$$

where we have used (13) in the equaltiy of (41) and (42), and the fact that $\left(\widehat{Z}_{t} \widehat{X}_{t}\right)_{0 \leq t \leq T}$ is a uniformly integrable martingale in the equalities of (40) and (41) as well as of (42) and (43).

As regards the final inequality, we used (35) and $\lim _{n \rightarrow \infty} \mathbf{E}\left[Z_{\tau_{n}} \mathbf{1}_{A_{n}}\right]=$ $\lim _{n \rightarrow \infty} Q\left[A_{n}\right]=0$, for every sequence $\left(A_{n}\right)_{n=1}^{\infty}$ of $\mathcal{F}_{\tau_{n}}$-measureable sets decreasing a.s. to the empty set.

We now aboard the proof of Proposition 1 for general utility functions $U: \mathbb{R} \rightarrow \mathbb{R}$ having reasonable asymptotic elasticity, i.e., satisfying (1) and (2). We shall develop a dynamic version of the utility maximisation problem, following the classical lines of control theory, as presented, e.g., in [E 81]. The subsequent presentation has greatly benefitted from detailed comments of an anonymous referee which are gratefully acknowledged.

Dynamic version of the utility maximisation problem. Given a stopping time $\tau$, valued in $[0, T]$, together with an arbitrary $\mathcal{F}_{\tau}$-measurable $\mathbb{R}$-valued random variable $\xi$, define the maximisation problem:

$$
u_{\tau}(\xi):=\operatorname{ess} \sup \mathbf{E}\left[U\left(\xi+\int_{\tau}^{T} H_{r} d S_{r}\right) \mid \mathcal{F}_{\tau}\right],
$$

where $H$ runs through the admissible integrands supported by the stochastic interval $\rrbracket \tau, T \rrbracket$.

Let us verify that $u_{\tau}(\xi)$ is a well-defined $\mathcal{F}_{\tau}$-measurable function (strictly speaking: an equivalence class of $\mathcal{F}_{\tau}$-measurable functions), taking values in the interval $[U(\xi), U(\infty)]$ almost surely. By considering $H=0$ we clearly have $u_{\tau}(\xi) \geq U(\xi)$. Fixing an arbitrary admissible integrand $H=H \mathbf{1}_{\rrbracket \tau, T \rrbracket}$, there is $C>0$ such that $(H \cdot S)_{t} \geq-C$ a.s., for all $t \in[0, T]$. Hence on the $\mathcal{F}_{\tau}$-measurable sets $\{\xi \geq-M\}$ we have $\xi+(H \cdot S)_{T}=\xi+\int_{\tau}^{T} H_{r} d S_{r} \geq-(M+C)$. Sending $M$ to infinity we conclude that, for fixed $H$, the conditional expectation in (44) is a well-defined (equivalence class of) $\mathcal{F}_{\tau}$-measureable function(s) taking values in $\left.]-\infty, U(\infty)\right]$. Hence the essential supremum defined in (44) is well-defined too, and $u_{\tau}(\xi)$ takes its values in $[U(\xi), U(\infty)]$ almost surely. 
We still note that one may deduce from Assumption 1 the more precise result that $u_{\tau}(\xi)$ takes its values in the interval $[U(\xi), U(\infty)$ [ a.s.; as we shall not need this sharpening we don't elaborate on its proof.

We now pass to the dynamic programming formula, which reads as follows: for any pair $\tau \leq \vartheta$ of $[0, T]$-valued stopping times we have

$$
u_{\tau}(\xi)=\operatorname{ess} \sup \mathbf{E}\left[u_{\vartheta}\left(\xi+\int_{\tau}^{\vartheta} H_{r} d S_{r}\right) \mid \mathcal{F}_{\tau}\right] .
$$

Here again $H$ runs through the admissible integrands supported by $\rrbracket \tau, T \rrbracket$ (or, equivalently, by $\rrbracket \tau, \vartheta \rrbracket)$ and $\xi$ is an $\mathcal{F}_{\tau}$-measurable $\mathbb{R}$-valued function.

To verify (45) we may assume that $\xi$ is bounded as it suffices to show (45) on the $\mathcal{F}_{\tau}$-measurable sets $\{-M \leq \xi \leq M\}$. To show the " $\geq$ " in (45), fix admissible integrands $H$ and $L$ supported by $\rrbracket \tau, T \rrbracket$ and $\rrbracket \vartheta, T \rrbracket$ respectively. Then $\widetilde{H}=H_{\rrbracket \tau, \vartheta \rrbracket}+L$ is an admissible integrand supported by $\rrbracket \tau, T \rrbracket$ so that

$$
u_{\tau}(\xi) \geq \mathbf{E}\left[U\left(\xi+\int_{\tau}^{\vartheta} H_{r} d S_{r}+\int_{\vartheta}^{T} L_{r} d S_{r}\right) \mid \mathcal{F}_{\tau}\right] .
$$

Using the definition of $u_{\vartheta}$ we obtain the inequality " $\geq$ " in (45).

To show the " $\leq$ " in (45), fix again an admissible integrand $H$ supported by $\rrbracket \tau, T \rrbracket$. For fixed $n$, the integrand $L^{n}:=H \mathbf{1}_{\rrbracket \vartheta, T \rrbracket} \mathbf{1}_{\left\{(H: S)_{\vartheta} \leq n\right\}}$ is admissible and supported by $\rrbracket \vartheta, T \rrbracket$, so that

$$
\begin{aligned}
\mathbf{E}\left[U\left(\xi+\int_{\tau}^{T} H_{r} d S_{r}\right) \mid \mathcal{F}_{\tau}\right] & \leq \sup _{n} \mathbf{E}\left[U\left(\xi+\int_{\tau}^{\vartheta} H_{r} d S_{r}+\int_{\vartheta}^{T} L_{r}^{n} d S_{r}\right) \mid \mathcal{F}_{\tau}\right] \\
& \leq \mathbf{E}\left[u_{\vartheta}\left(\xi+\int_{\tau}^{\vartheta} H_{r} d S_{r}\right) \mid \mathcal{F}_{\tau}\right] .
\end{aligned}
$$

Dynamic version of the dual problem. Given an arbitrary stopping time $\tau$ valued in $[0, T]$ together with an $\mathcal{F}_{\tau}$-measurable strictly positive random variable $\zeta$, define

$$
\mathcal{Z}_{\tau}^{S}(\zeta):=\left\{Y:=\zeta \frac{d \mathbf{Q} / d \mathbf{P}}{\mathbf{E}\left[(d \mathbf{Q} / d \mathbf{P}) \mid \mathcal{F}_{\tau}\right]}: \mathbf{Q} \in \mathcal{M}^{e}(S)\right\}
$$

and the associated minimisation problem:

$$
v_{\tau}(\zeta):=\operatorname{essinf}_{Y \in \mathcal{Z}_{\tau}^{S}(\zeta)} \mathbf{E}\left[V(Y) \mid \mathcal{F}_{\tau}\right]
$$

Using Assumption 1 and similar arguments as in [BF 02] or [S 01] one verifies that any minimizing $\left(Y^{n}\right)_{n=1}^{\infty}$ converges a.s. to an element $\widehat{Y}(\zeta) \in \mathcal{Z}_{\tau}^{S}(\zeta)$ for which we have

$$
v_{\tau}(\zeta)=\mathbf{E}\left[V(\widehat{Y}(\zeta)) \mid \mathcal{F}_{\tau}\right]
$$

Also note that $v_{\tau}(\zeta)$ is a.s. finite and that $\zeta \mapsto v_{\tau}(\zeta)$ is a convex function.

The controls set $\mathcal{Z}_{\tau}^{S}(\zeta)$ is closed under the concatenation property described in Lemma 2. This implies, similarly as (but somewhat easier than) above, the dynamic programming formula:

$$
v_{\tau}(\zeta)=\underset{Y \in \mathcal{Z}_{\tau}^{S}(\zeta)}{\operatorname{essinf}} \mathbf{E}\left[v_{\vartheta}\left(Y_{\vartheta}\right) \mid \mathcal{F}_{\tau}\right] \text { with } Y_{\vartheta}:=\mathbf{E}\left[Y \mid \mathcal{F}_{\vartheta}\right]
$$


for any pair of $[0, T]$-valued stopping times $\tau \leq \vartheta$.

We may also define the right hand side derivative $v_{\tau}^{\prime}$ of the conditional value function $v_{\tau}$ by

$$
v_{\tau}^{\prime}(\zeta)=\lim _{\lambda \searrow 1} \frac{v_{\tau}(\lambda \zeta)-v_{\tau}(\zeta)}{\zeta(\lambda-1)}
$$

Note that the right hand side is decreasing in $\lambda$ and bounded from below by $\frac{v_{\tau}(\zeta)-v_{\tau}(\zeta / 2)}{\zeta / 2}$, so that $v_{\tau}^{\prime}(\zeta)$ is a well-defined, finitely valued (equivalence class of) $\mathcal{F}_{\tau^{-}}$ measurable function(s). We also observe that $\zeta_{1} \leq \zeta_{2}$ implies $v_{\tau}^{\prime}\left(\zeta_{1}\right) \leq v_{\tau}^{\prime}\left(\zeta_{2}\right)$ almost surely.

One may, in fact, show that $v_{t}^{\prime}$ is a derivative, and not only a right hand side derivative (compare [S 01, Step 5 of the proof of Theorem 2.2]). But we want to develop here only the minimum of the conditional theory, which enables us to prove Proposition 1; Definition (52) is taylor-made to show the subsequent crucial estimate in an easy way.

Lemma 3 Under the assumptions of Theorem 1, also assume $U(0)>0$.

There is a constant $C>0$, depending only on the utility function $U$, such that, for every $[0, T]$-valued stopping time $\tau$ and every $\mathcal{F}_{\tau}$-measurable $\zeta>0$, we have

$$
\zeta v_{\tau}^{\prime}(\zeta) \leq C v_{\tau}(\zeta), \quad \text { a.s. }
$$

Proof We apply [S 01, Corollary 4.2 (ii)] to find $C>0$ such that $y V^{\prime}(y) \leq C V(y)$, for all $y>0$. Hence

$$
\begin{aligned}
\zeta v_{\tau}^{\prime}(\zeta) & =\lim _{\lambda \searrow 1} \mathbf{E}\left[V(\widehat{Y}(\lambda \zeta))-V(\widehat{Y}(\zeta)) \mid \mathcal{F}_{\tau}\right] /(\lambda-1) \\
& \leq \lim _{\lambda \searrow 1} \mathbf{E}\left[V(\lambda \widehat{Y}(\zeta))-V(\widehat{Y}(\zeta)) \mid \mathcal{F}_{\tau}\right] /(\lambda-1) \\
& \leq \lim _{\lambda \searrow 1} \mathbf{E}\left[\widehat{Y}(\zeta) V^{\prime}(\lambda \widehat{Y}(\zeta)) \mid \mathcal{F}_{\tau}\right]=\lim _{\lambda \searrow 1} \mathbf{E}\left[\lambda \widehat{Y}(\zeta) V^{\prime}(\lambda \widehat{Y}(\zeta)) \mid \mathcal{F}_{\tau}\right] \\
& \leq \lim _{\lambda \searrow 1} C \mathbf{E}\left[V(\lambda \widehat{Y}(\zeta)) \mid \mathcal{F}_{\tau}\right]=C \mathbf{E}\left[V(\widehat{Y}(\zeta)) \mid \mathcal{F}_{\tau}\right]=C v_{\tau}(\zeta),
\end{aligned}
$$

where (54) follows from Lebesgue's theorem and [S 01, Corollary 4.2 (i)].

We also need conditional versions of some of the results from [KrS 99] and [S 01], which are resumed in the subsequent lemma.

Lemma 4 Under the assumptions of Theorem 1 , let $x \in \mathbb{R}, y=u^{\prime}(x)$, denote by $\widehat{H}(x)$ and $\widehat{\mathbf{Q}}(y)$ the primal and the dual optimizer, by $\left(\widehat{Z}_{t}(y)\right)_{0 \leq t \leq T}$ the density process of $\widehat{\mathbf{Q}}(y)$. Fix a $[0, T]$-valued stopping time $\tau$. Letting $\xi=x+(\widehat{H}(x) \cdot S)_{\tau}$ and $\zeta=y \widehat{Z}_{\tau}(y)$ we have the following properties:

(i) The optimal values in (44) and (49) are attained by $H:=\widehat{H}(x) \mathbf{1}_{\rrbracket \tau, T \rrbracket}$ and $Y:=$ $y \widehat{Z}_{T}(y)$ respectively. 
(ii) The following duality relation holds true almost surely:

$$
-v_{\tau}^{\prime}(\zeta)=\xi
$$

Proof (i) As regards the first assertion, things are slightly subtle, as neither $\widehat{H}(x)$ nor $\widehat{H}(x) \mathbf{1}_{\rrbracket \tau, T \rrbracket}$ is necessarily admissible. To alleviate notation we write $\widehat{H}$ for $\widehat{H}(x)$.

We first show the inequality

$$
u_{\tau}(\xi) \leq \mathbf{E}\left[U\left(\xi+\int_{\tau}^{T} \widehat{H}_{r} d S_{r}\right) \mid \mathcal{F}_{\tau}\right]
$$

Indeed, suppose there is an admissible integrand $H=H \mathbf{1}_{\rrbracket \tau, T \rrbracket}$ and a set $A \in \mathcal{F}_{\tau}$, $\mathbf{P}[A]>0$, such that

$$
\mathbf{E}\left[U\left(\xi+\int_{\tau}^{T} H_{r} d S_{r}\right) \mathbf{1}_{A}\right]>\mathbf{E}\left[U\left(\xi+\int_{\tau}^{T} \widehat{H}_{r} d S_{r}\right) \mathbf{1}_{A}\right]
$$

For $\tau^{A}:=\tau \mathbf{1}_{A}+T \mathbf{1}_{\Omega \backslash A}$ and $\widetilde{H}:=\widehat{H}+(H-\widehat{H}) \mathbf{1}_{\rrbracket \tau^{A}, T \rrbracket}$, we have

$$
\mathbf{E}\left[U\left(x+\int_{0}^{T} \widetilde{H}_{r} d S_{r}\right)\right]>\mathbf{E}\left[U\left(x+\int_{0}^{T} \widehat{H}_{r} d S_{r}\right)\right] .
$$

Note that $\widetilde{H} \cdot S$ is a $\widehat{\mathbf{Q}}(y)$-supermartingale, hence by the usual Fenchel duality argument (see (17), (18) above) inequality (58) is in contradiction to the optimality of $\widehat{H}$, which proves $(56)$.

To show the converse inequality

$$
u_{\tau}(\xi) \geq \mathbf{E}\left[U\left(\xi+\int_{\tau}^{T} \widehat{H}_{r} d S_{r}\right) \mid \mathcal{F}_{\tau}\right] .
$$

we use the fact ([S 01, Theorem 2.2]) that $\widehat{H}$ can be approximated by a sequence of admissible integrands in the sense of Definition 3 (iii). Using Proposition 5 from the Appendix we may find, for $\epsilon>0$, an admissible integrand $H^{\epsilon}$ such that

$$
\mathbf{E}\left[U\left(x+\left(H^{\epsilon} \cdot S\right)_{T} \wedge(\widehat{H} \cdot S)_{T}\right)\right]>\mathbf{E}\left[U\left(x+(\widehat{H} \cdot S)_{T}\right)\right]-\epsilon,
$$

and

$$
\mathbf{P}\left[\xi>x+\left(H^{\epsilon} \cdot S\right)_{\tau}-\epsilon\right]>1-\epsilon .
$$

(Note that $\left.\xi=x+(\widehat{H} \cdot S)_{\tau}\right)$. Define $A^{\epsilon}$ to be the $\mathcal{F}_{\tau}$-measurable set

$$
A^{\epsilon}=\left\{\xi>x+\left(H^{\epsilon} \cdot S\right)_{\tau}-\epsilon,\left(H^{\epsilon} \cdot S\right)_{\tau}<\epsilon^{-1}\right\}
$$

and $\tau^{A^{\epsilon}}=\tau \mathbf{1}_{A^{\epsilon}}+T \mathbf{1}_{\Omega \backslash A^{\epsilon}}$. The process $\widetilde{H}^{\epsilon}=H^{\epsilon} \mathbf{1}_{\rrbracket \tau^{A^{\epsilon}}, T \rrbracket}$ is an admissible integrand supported by $\rrbracket \tau, T \rrbracket$ and it is straightforward to verify that

$$
u_{\tau}(\xi) \geq \underset{\epsilon>0}{\operatorname{ess} \sup _{0}} \mathbf{E}\left[U\left(\xi+\int_{\tau}^{T} \widetilde{H}_{r}^{\epsilon} d S_{r}\right) \mid \mathcal{F}_{\tau}\right] \geq \mathbf{E}\left[U\left(\xi+\int_{\tau}^{T} \widehat{H}_{r} d S_{r}\right) \mid \mathcal{F}_{\tau}\right] .
$$


The verification that $Y:=y \widehat{Z}_{\tau}(y)$ yields the optimal value in (49) is easier and left to the reader.

(ii) is simply the conditional version of [S 01, Theorem 2.2 (iv)]:

$$
\begin{aligned}
\xi & =\mathbf{E}_{\widehat{\mathbf{Q}}(y)}\left[x+(\widehat{H} \cdot S)_{T} \mid \mathcal{F}_{\tau}\right]=-\mathbf{E}_{\widehat{\mathbf{Q}}(y)}\left[V^{\prime}\left(y \widehat{Z}_{T}(y)\right) \mid \mathcal{F}_{\tau}\right] \\
& =-\mathbf{E}_{\mathbf{P}}\left[\widehat{Z}_{T}(y) V^{\prime}\left(y \widehat{Z}_{T}(y)\right) \mid \mathcal{F}_{\tau}\right]=-v_{\tau}^{\prime}(\zeta) .
\end{aligned}
$$

We have used in the first equality the $\widehat{\mathbf{Q}}(y)$-martingale property of $\widehat{H} \cdot S$, and in the second [S 01, Theorem 2.2(iv)]. The final equality is the conditional version of [S 01, Theorem $2.2(\mathrm{v})$ ] and can be shown similarly as Lemma 3 above (compare the proof of $[\mathrm{KrS} 99$, Theorem 2.2]).

\section{Proof of Proposition 1 for the general case}

First note that there is no loss of generality in assuming that $U(0)>0$, as the assertion is clearly invariant under adding a constant to $U$. Suppose that there is $\mathbf{Q} \in \mathcal{M}^{a}(S)$, $\mathbf{E}\left[V\left(\frac{d \mathbf{Q}}{d \mathbf{P}}\right)\right]<\infty$, and $x \in \mathbb{R}$, such that $\widehat{X}(x)$ fails to be a $\mathbf{Q}$-super-martingale.

Similarly as in (35) above we conclude from Lemma 1 that there is a sequence $\left(\tau_{n}\right)_{n=1}^{\infty}$ of stopping times, increasing to $\infty$, such that

$$
\liminf _{n \rightarrow \infty} \mathbf{E}_{\mathbf{P}}\left[-\widehat{X}_{\tau_{n}}(x) Y_{\tau_{n}} \mathbf{1}_{\left\{\tau_{n}<\infty, Y_{\tau_{n}} \geq \widehat{Y}_{\tau_{n}}\right\}}\right]>0,
$$

where $Y_{t}$ is the density process of $y \frac{d \mathbf{Q}}{d \mathbf{P}}, \widehat{Y}_{t}$ the density process of $y \frac{d \widehat{\mathbf{Q}}(y)}{d \mathbf{P}}$ and $u^{\prime}(x)=y$.

Applying Lemma 3 and 4 to the stopping time $\tau_{n} \wedge T$ and letting $c=C^{-1}$, we then compute that:

$$
\begin{aligned}
\mathbf{E}_{\mathbf{P}}\left[V(Y) \mathbf{1}_{\left\{\tau_{n}<\infty, Y_{\tau_{n}} \geq \widehat{Y}_{\tau_{n}}\right\}}\right] & =\mathbf{E}_{\mathbf{P}}\left[\mathbf{E}\left(V(Y) \mid \mathcal{F}_{\tau_{n}}\right) \mathbf{1}_{\left\{\tau_{n}<\infty, Y_{\tau_{n}} \geq \widehat{Y}_{\tau_{n}}\right\}}\right] \\
& \geq \mathbf{E}_{\mathbf{P}}\left[v_{\tau_{n}}\left(Y_{\tau_{n}}\right) \mathbf{1}_{\left\{\tau_{n}<\infty, Y_{\left.\tau_{n} \geq \widehat{Y}_{\tau_{n}}\right\}}\right]}\right.
\end{aligned}
$$

by definition of the minimization problem $v_{\tau_{n}}$

$$
\geq c \mathbf{E}_{\mathbf{P}}\left[Y_{\tau_{n}} v_{\tau_{n}}^{\prime}\left(Y_{\tau_{n}}\right) \mathbf{1}_{\left\{\tau_{n}<\infty, Y_{\tau_{n}} \geq \widehat{Y}_{\tau_{n}}\right\}}\right]
$$

by Lemma 3

$$
\geq c \mathbf{E}_{\mathbf{P}}\left[Y_{\tau_{n}} v_{\tau_{n}}^{\prime}\left(\widehat{Y}_{\tau_{n}}\right) \mathbf{1}_{\left\{\tau_{n}<\infty, Y_{\tau_{n}} \geq \widehat{Y}_{\tau_{n}}\right\}}\right]
$$

by the monotonicity of $v_{\tau_{n}}^{\prime}(\cdot)$

$$
=c \mathbf{E}_{\mathbf{P}}\left[-Y_{\tau_{n}} \widehat{X}_{\tau_{n}} \mathbf{1}_{\left\{\tau_{n}<\infty, Y_{\tau_{n}} \geq \widehat{Y}_{\tau_{n}}\right\}}\right]
$$

by Lemma 4 .

Sending $n$ to $\infty$, we deduce from (64) a contradiction to the $\mathbf{P}$-integrability of $V(Y)$ and the fact that $\left(\tau_{n}\right)_{n=1}^{\infty}$ increases to $\infty$. 


\section{The Role of Potential Investments: a Puzzling Example}

In this section we shall construct some examples, which illustrate the precise limits of Theorem 1.

The most important example is summarized in Proposition 2 below. It highlights the delicacy of the "good definition" of "allowed" trading strategies. It has a surprising economic interpretation, to which we tried to refer to in the title of the present section.

It will be notationally convenient to take as time index set $\mathbb{N}_{0} \cup\{\infty\}$ instead of $[0, T]$; by replacing $\mathbb{N}_{0} \cup\{\infty\}$ by $\left(t_{n}\right)_{n=0}^{\infty} \cup\{T\}$, where $0=t_{0}<t_{1}<\ldots<t_{n}<\ldots$ is a sequence increasing to $T$, it is obvious how to translate the present result into the framework of the previous section (compare also [S 02]).

Proposition 2 There are processes $S^{1}=\left(S_{n}^{1}\right)_{n \in \mathbb{N}_{0}}$ and $S^{2}=\left(S_{n}^{2}\right)_{n \in \mathbb{N}_{0}}$, defined on and adapted to $\left(\Omega, \mathcal{F},\left(\mathcal{F}_{n}\right)_{n \in \mathbb{N}_{0}}, \mathbf{P}\right)$, and a probability measure $\mathbf{Q} \sim \mathbf{P}$ on $\mathcal{F}$, with the following properties:

(i) $H(\mathbf{Q} \mid \mathbf{P})<\infty$.

(ii) $S^{1}$ is a martingale under $\mathbf{Q}$ and $\mathbf{P}$, which is uniformly integrable under $\mathbf{Q}$, but not uniformly integrable under $\mathbf{P}$.

(iii) $S^{2}$ is a martingale under $\mathbf{Q}$, but not a martingale under $\mathbf{P}$.

(iv) Denoting by $S^{\text {large }}$ the $\mathbb{R}^{2}$-valued process $\left(S^{1}, S^{2}\right), \mathbf{Q}$ is the unique equivalent local martingale measure for $S^{\text {large }}$. The equality

$$
S_{\infty}^{1}=-\ln \left(\frac{d \mathbf{Q}}{d \mathbf{P}}\right)
$$

holds true, and therefore the process $\left(S_{n}^{1}\right)_{n \in \mathbb{N}_{0}}$ equals the investment process $\left(\widehat{X}_{n}\right)_{n \in \mathbb{N}_{0}}$, starting at $\widehat{X}_{0}=S_{0}^{1}$, which is optimal with respect to the utility function $U(x)=-e^{-x}$.

(v) Denoting by $S^{\text {small }}$ the $\mathbb{R}$-valued process $\left(S_{n}^{1}\right)_{n \in \mathbb{N}_{0}}, \mathbf{P}$ is a martingale measure for $S^{\text {small }}$. Hence the optimal investment process with initial endowment $\widehat{X}_{0}=S_{0}^{1}$ now is the constant process $\widehat{X}_{n} \equiv S_{0}^{1}$. The optimality pertains to $U(x)=-e^{-x}$ (and, in fact, to any $U$ satisfying (1)).

Let us briefly comment on this result: In the case of the "large" financial market $S^{\text {large }}$, the optimal investment consists in constantly holding one unit of the first asset $S^{1}$ and not touching the second asset $S^{2}$ (and also not using any information revealed by $S^{2}$ ). Nevertheless, by passing to the "small" financial market $S^{\text {small }}$, which consists of this first asset only, defined over the same filtered stochastic base $\left(\Omega, \mathcal{F},\left(\mathcal{F}_{n}\right)_{n \in \mathbb{N}_{0}}, \mathbf{P}\right)$, this strategy is not optimal any more! In this case the optimal strategy is not to invest into the risky asset, and to keep the money in the bond.

Hence the role of the asset $S^{2}$ may be compared to a catalyst in chemistry: its sheer presence changes the situation without entering into the chemical reaction. 
One reason for this seemingly paradoxical result is that in the former case the optimal strategy $H=(1,0)$ of holding one unit of the first asset can be approximated by a sequence $H^{n}=\left(H^{n, 1}, H^{n, 2}\right)$ of admissible strategies in the sense of Definition 3 (iii) above. The second asset is needed for these approximating strategies and such an approximation is not possible by only trading in the first asset.

We postpone the proof of Proposition 2 and resume two more examples. Proposition 3 shows that in Proposition 1 above the finiteness of $H(\mathbf{Q} \mid \mathbf{P})$, or, more generally, of $E\left[V\left(\frac{d \mathbf{Q}}{d \mathbf{P}}\right)\right]$, cannot be dropped.

Proposition 3 There is an $\mathbb{R}$-valued financial market $\left(S_{n}\right)_{n \in \mathbb{N}_{0}}$, such that the entropyminimal element $\widehat{\mathbf{Q}} \in \mathcal{M}^{e}(S)$ as well as the optimal investment process $\widehat{X}(0)$ with respect to $U(x)=-e^{-x}$ exist, and there is some equivalent martingale measure $\mathbf{Q} \in$ $\mathcal{M}^{e}(S)$, with $H(\mathbf{Q} \mid \mathbf{P})=\infty$, for which the closed process $\widehat{X}(0)=\left(\widehat{X}_{n}(0)_{n \in \mathbb{N}_{0} \cup\{\infty\}}\right)$ is only a local $\mathbf{Q}$-martingale, but not a $\mathbf{Q}$-super-martingale.

Finally we show that the last assertion of Theorem 1 for the exponential utility $U(x)=-e^{-x}$, does not extend to general utility functions $U: \mathbb{R} \rightarrow \mathbb{R}$.

Proposition 4 There is a utility function $U: \mathbb{R} \rightarrow \mathbb{R}$ satisfying (1) and (2), and an $\mathbb{R}$-valued financial market $\left(S_{n}\right)_{n \in \mathbb{N}_{0}}$, such that there is $\mathbf{Q} \in \mathcal{M}^{e}(S)$ with finite $V$ expectation $\mathbf{E}\left[V\left(\frac{d \mathbf{Q}}{d \mathbf{P}}\right)\right]<\infty$ and $x_{0} \in \mathbb{R}$, such that the closed optimal process $\widehat{X}\left(x_{0}\right)=$ $\left(\widehat{X}_{n}\left(x_{0}\right)\right)_{n \in \mathbb{N}_{0} \cup\{\infty\}}$ fails to be a $\mathbf{Q}$-submartingale.

The construction of all three counter-examples will follow the same pattern which goes back to the construction of [S 93] and which we now describe. This presentation has greatly benefitted from detailed comments by Y. Kabanov [Ka 02], which are gratefully acknowledged.

Consider the fair game consisting of a sequence of independent trials (binary experiments) where in the $k^{\text {th }}$-trial the increment of the player's capital is $y_{k}>0$ or $x_{k}<0$, with the "success" probability being $\left.l_{k} \in\right] 0,1[$. The player also observes another independent sequence of binary experiments with success probabilities $\left.p_{k} \in\right] 0,1$ [; the game stops at the first success in any of these two sequences.

We formalize this in a standard way. For $k \geq 1$, let $\Omega_{k}^{\prime}, \Omega_{k}^{\prime \prime}$ be the "elementary" probability spaces $\{-1,1\}$ with $\mathbf{P}^{\prime}(\{1\})=l_{k}, \mathbf{P}^{\prime \prime}(\{1\})=p_{k}$ (for the binary experiments) and let $(\Omega, \mathcal{F}, \mathbf{P})$ be the product of all these spaces. For $\omega=\left(\omega^{\prime}, \omega^{\prime \prime}\right) \in \Omega$ we take $\eta_{k}(\omega)=y_{k}$ or $x_{k}$ in dependence whether $\omega_{k}^{\prime}=1$ or $\omega_{k}^{\prime}=-1$, and $\xi_{k}(\omega)=\omega_{k}^{\prime \prime}$.

We assume that $\mathbf{E}\left[\eta_{k}\right]=0$. In this case, for any initial value $a_{0} \in \mathbb{R}$, the random walk $\Sigma_{n}:=a_{0}+\sum_{k=1}^{n} \eta_{k}$ is a martingale with respect to the filtration $\left(\mathcal{F}_{n}^{\xi, \eta}\right)_{n=0}^{\infty}$.

Define the stopping times $\tau=\inf \left\{n: \eta_{n}>0\right\}$ and $\sigma=\inf \left\{n: \xi_{n}>0\right\}$.

The stopped process $S:=\Sigma^{\tau \wedge \sigma}$ is a martingale with respect to $\left(\mathcal{F}_{n}^{\xi, \eta}\right)_{n=0}^{\infty}$ and, of course, with respect to its natural filtration $\left(\mathcal{F}_{n}^{S}\right)_{n=0}^{\infty}$.

Put $a_{n}=a_{0}+\sum_{k=1}^{n} x_{k}$ and $b_{n}=y_{n}+a_{n-1}$. The sequences $\left(a_{n}\right)_{n=0}^{\infty},\left(b_{n}\right)_{n=1}^{\infty}$ can be taken as the primary parameters of the model. We assume that $b_{n} \geq 0, a_{n} \leq 0$, and $a_{n} \rightarrow-\infty$. 
Note that the condition $\mathbf{E}\left[\eta_{n}\right]=0$ means that

$$
l_{n}=\mathbf{P}\left(\eta_{n}>0\right)=\frac{a_{n-1}-a_{n}}{b_{n}-a_{n}}, \quad 1-l_{n}=\mathbf{P}\left(\eta_{n}<0\right)=\frac{b_{n}-a_{n-1}}{b_{n}-a_{n}} .
$$

Put also $e_{n}=\Pi_{j \leq n}\left(1-l_{j}\right)$.

Let $C_{0}=\Omega$ and define, for $n \geq 1$, the sets

$$
A_{n}=\{\sigma=n<\tau\}, B_{n}=\{\tau=n \leq \sigma\}, C_{n}=\left\{S_{n}=a_{n}\right\} .
$$

The interpretation goes as follows: the set $C_{n}$, which may equivalently be defined as $C_{n}=\{\tau>n, \sigma \geq n\}$ equals the set where the game has continued up to time $n$ and the gambler has been losing all $n$ games, so that $S_{n}=a_{n}=a_{0}+\sum_{k=1}^{n} x_{k}$ on $C_{n}$. For $n \geq 1$, the set $C_{n}$ is split into the three sets $A_{n}, B_{n+1}, C_{n+1}$ : on $A_{n}=C_{n} \cap\left\{\omega_{n}^{\prime \prime}=1\right\}$ the game stops at time $n$, as the observation of $\xi_{n}(\omega)=\omega_{n}^{\prime \prime}$ tells the gambler to do so; on $B_{n+1}=C_{n} \cap\left\{\omega_{n}^{\prime \prime}=-1, \omega_{n+1}^{\prime}=1\right\}$ the gambler continues to play the $(n+1)^{\text {th }}$ round and wins (so that she stops at time $n+1$ ); finally on $C_{n+1}=C_{n} \cap\left\{\omega_{n}^{\prime \prime}=\omega_{n}^{\prime}=-1\right\}$ the gambler continues to play the $(n+1)^{\text {th }}$ round and loses.

Clearly, the $\sigma$-algebra $\mathcal{F}_{n}^{S}$ is generated by the partition $A_{1}, \ldots, A_{n-1}, B_{1}, \ldots, B_{n}$, $C_{n}$. Also note that $S_{n}$ (as well as $S_{m}$, for $m \geq n$ ) equals $\left(a_{k}\right)_{k=1}^{n}$ and $\left(b_{k}\right)_{k=1}^{n}$ on the sets $\left(A_{k}\right)_{k=1}^{n}$ and $\left(B_{k}\right)_{k=1}^{n}$ respectively.

The random variable $\zeta_{n+1}:=\mathbf{1}_{\left\{\xi_{n}=1\right\}} \mathbf{1}_{C_{n}}$ is $\mathcal{F}_{n+1}^{S}$ measurable. Since $\xi_{n}$ and $\mathcal{F}_{n}^{S}$ are independent, $\mathbf{E}\left[\zeta_{n+1} \mid \mathcal{F}_{n}^{S}\right]=p_{n} \mathbf{1}_{C_{n}}$. Thus, if $\mathbf{Q}$ is a measure on $(\Omega, \mathcal{F})$ defined in the same way as $\mathbf{P}$, but with $p_{k}$ replaced by $\left.q_{k} \in\right] 0,1[$,

$$
S_{n}^{2}:=\sum_{k=1}^{n} 2^{-k}\left(\zeta_{k+1}-q_{k} \mathbf{1}_{C_{k}}\right), \quad n \geq 0,
$$

is a bounded Q-martingale with respect to $\left(\mathcal{F}_{n}^{S}\right)_{n=0}^{\infty}$.

Lemma 5 If $\widetilde{\mathbf{Q}}$ is a measure on $\mathcal{F}_{\infty}^{S}$ such that the $\mathbb{R}^{2}$-valued process $S^{\text {large }}=\left(S, S^{2}\right)$ is a $\widetilde{\mathbf{Q}}$-martingale, then $\widetilde{\mathbf{Q}}=\mathbf{Q}^{S}$ where $\mathbf{Q}^{S}:=\left.\mathbf{Q}\right|_{\mathcal{F}_{\infty}^{S}}$.

Proof We proceed by induction and suppose that we have proved the equality of $\widetilde{\mathbf{Q}}$ and $\mathbf{Q}^{S}$ on $\mathcal{F}_{n}^{S}$ so that in particular $\widetilde{\mathbf{Q}}\left[C_{n}\right]=\mathbf{Q}^{S}\left[C_{n}\right]$. By the martingale property $E_{\widetilde{\mathbf{Q}}}\left[\mathbf{1}_{C_{n}} \Delta S_{n+1}\right]=0$ and $E_{\widetilde{\mathbf{Q}}}\left[\mathbf{1}_{C_{n}} \Delta S_{n+1}^{2}\right]=0$, or

$$
x_{n+1} \widetilde{\mathbf{Q}}\left(C_{n+1}\right)+y_{n+1} \tilde{\mathbf{Q}}\left(B_{n+1}\right)=0, \quad \widetilde{\mathbf{Q}}\left(A_{n}\right)-q_{n} \widetilde{\mathbf{Q}}\left(C_{n}\right)=0 .
$$

Since similar relations hold for $\mathbf{Q}^{S}$, both measures coincide also on the sets $A_{n}$, $B_{n+1}$, and $C_{n+1}$ ("splitting" $C_{n}$ ), hence, on $\mathcal{F}_{n+1}^{S}$.

Lemma 6 Suppose that $S$ is $L^{1}$-bounded and

$$
0<\limsup _{n}\left|a_{n}\right| e_{n}<\infty .
$$

Then $S$ is uniformly integrable under $\mathbf{P}$ iff $\sum p_{n}=\infty$. 
Proof Recall that $\left|a_{n}\right| \rightarrow \infty$. Thus, (75) implies that $e_{n} \rightarrow 0$ (hence $\tau<\infty$ a.s.) and $\mathbf{P}\left(C_{n}\right)=e_{n} \Pi_{k<n}\left(1-p_{k}\right) \rightarrow 0$. If $S$ is uniformly integrable, then

$$
\lim _{n \rightarrow \infty}\left|a_{n}\right| e_{n} \Pi_{k<n}\left(1-p_{k}\right)=\lim _{n \rightarrow \infty} \mathbf{E}\left[\left|S_{n}\right| \mathbf{1}_{C_{n}}\right]=0 .
$$

Under condition (75) this is possible only if $\sum_{k} p_{k}=\infty$. On the other hand, the divergence of $\sum p_{n}$ and (75) imply (76). Hence,

$$
\mathbf{E}\left[\left|S_{\infty}-S_{n}\right|\right]=\mathbf{E}\left[\left|S_{\infty}-S_{n}\right| \mathbf{1}_{C_{n}}\right] \leq \mathbf{E}\left[\left|S_{\infty}\right| \mathbf{1}_{C_{n}}\right]+\mathbf{E}\left[\left|S_{n}\right| \mathbf{1}_{C_{n}}\right] \rightarrow 0,
$$

$\left(\mathbf{E}\left[\left|S_{\infty}\right|\right]<\infty\right.$ due to $L^{1}$-boundedness $)$, and $S$ is uniformly integrable.

Lemma 7 The process $S$ is $L^{1}$-bounded if either

$$
C:=\sup _{n} b_{n}<\infty
$$

or

$$
\sum_{n=1}^{\infty} \frac{b_{n}}{\left|a_{n-1}\right|}<\infty .
$$

Proof If (78) holds true, the martingale $S$ is bounded from above by $C$, hence $L^{1}$ bounded: $\mathbf{E}\left[\left|S_{n}\right|\right] \leq C+\mathbf{E}\left[\left|S_{n}-C\right|\right]=2 C$.

Now suppose that (79) holds true and estimate (using $a_{n} \leq a_{n-1} \leq 0 \leq b_{n}$ and the martingale property of $S$ ):

$$
\begin{aligned}
& \left\|S_{n}\right\|_{L^{1}(\mathbf{P})}-\left\|S_{n-1}\right\|_{L^{1}(\mathbf{P})}=\mathbf{E}\left[\left|S_{n} \mathbf{1}_{C_{n-1}}\right|\right]-\mathbf{E}\left[\left|S_{n-1} \mathbf{1}_{C_{n-1}}\right|\right] \\
& \quad=2 \mathbf{E}\left[S_{n} \mathbf{1}_{B_{n}}\right]=\frac{2 b_{n}}{\left|a_{n-1}\right|} \mathbf{E}\left[\left|S_{n-1} \mathbf{1}_{B_{n}}\right|\right] \leq \frac{2 b_{n}}{\left|a_{n-1}\right|}\left\|S_{n-1}\right\|_{L^{1}(\mathbf{P})} .
\end{aligned}
$$

From (79) we obtain the $L^{1}(\mathbf{P})$-boundedness of $S$.

Proof of Proposition 2 We define the process $S^{1}$ as the above process $S$ associated to the following sequences $\left(a_{n}\right)_{n=0}^{\infty},\left(b_{n}\right)_{n=1}^{\infty},\left(p_{n}\right)_{n=1}^{\infty},\left(q_{n}\right)_{n=1}^{\infty}$ :

$$
\begin{gathered}
p_{n}=e^{-2^{n}}, \quad q_{n}=\frac{1}{n+1}, \quad n \geq 1, \\
a_{n}:=\ln \left(n(n+1) p_{n} \prod_{j=1}^{n-1}\left(1-p_{j}\right)\right) \approx-2^{n}, \quad n \geq 1, \\
b_{n}:=\ln \left(n \prod_{j=1}^{n-1}\left(1-p_{j}\right)\right) \approx \ln (n), \quad n \geq 1,
\end{gathered}
$$

where we use the notations $\prod_{j=1}^{0}:=1$ and $\alpha_{n} \approx \beta_{n}$, if there are constants $0<c \leq$ $C<\infty$ such that $c \alpha_{n} \leq \beta_{n} \leq C \alpha_{n}$, for all $n \in \mathbb{N}$. Note that $a_{1}=\ln \left(2 e^{-2}\right)<0=b_{1}$ and choose $a_{0}$ to be an arbitrary element of $] a_{1}, b_{1}\left[\right.$. Note that $\left(a_{n}\right)_{n=0}^{\infty}$ decreases to $-\infty$, and (79) is satisfied. 
Observing that (71) gives $l_{n} \approx \frac{1}{2}$ and $e_{n} \approx 2^{-n}$, we obtain the subsequent quantities for the measures $\mathbf{Q}$ and $\mathbf{P}$ defined above:

$$
\begin{aligned}
& \mathbf{P}\left[A_{n}\right]=e_{n} p_{n} \prod_{j=1}^{n-1}\left(1-p_{j}\right) \approx 2^{-n} e^{-2^{n}}, \\
& \mathbf{P}\left[B_{n}\right]=e_{n-1} l_{n} \prod_{j=1}^{n-1}\left(1-p_{j}\right) \approx 2^{-n}
\end{aligned}
$$

and

$$
\begin{aligned}
& \mathbf{Q}\left[A_{n}\right]=e_{n} q_{n} \prod_{j=1}^{n-1}\left(1-q_{j}\right)=\frac{e_{n}}{n(n+1)} \approx \frac{2^{-n}}{n^{2}} \\
& \mathbf{Q}\left[B_{n}\right]=e_{n-1} l_{n} \prod_{j=1}^{n-1}\left(1-q_{j}\right)=\frac{e_{n-1} l_{n}}{n} \approx \frac{2^{-n}}{n} .
\end{aligned}
$$

Observe that we have arranged things in such a way that we have

$$
a_{n}=-\ln \left(\frac{\mathbf{Q}\left[A_{n}\right]}{\mathbf{P}\left[A_{n}\right]}\right), \quad b_{n}=-\ln \left(\frac{\mathbf{Q}\left[B_{n}\right]}{\mathbf{P}\left[B_{n}\right]}\right), \quad \text { for } n \geq 1 .
$$

As $S_{\infty}^{1}$ equals $a_{n}$ on $A_{n}$ and $b_{n}$ on $B_{n}$, this amounts to (70).

By Lemma 6 and $7, S^{1}=\left(S_{n}^{1}\right)_{n=0}^{\infty}$ is a martingale under $\mathbf{Q}$ and $\mathbf{P}$, which is uniformly integrable under $\mathbf{Q}$ but not under $\mathbf{P}$.

Define $\left(S_{n}^{2}\right)_{n=0}^{\infty}$ as in (73), so that $S^{2}$ is a bounded martingale under $\mathbf{Q}$, and by Lemma $5 \mathrm{Q}$ is the unique equivalent martingale measure for the process $S^{\text {large }}=$ $\left(S^{1}, S^{2}\right)$ on $\mathcal{F}_{\infty}^{S}$.

We now turn to the verification of assertions (i)-(v):

(i) We have, for a constant $C>0$ sufficiently large,

$$
\begin{aligned}
H(\mathbf{Q} \mid \mathbf{P}) & =\sum_{n=1}^{\infty}\left(\mathbf{Q}\left[A_{n}\right] \ln \left(\frac{\mathbf{Q}\left[A_{n}\right]}{\mathbf{P}\left[A_{n}\right]}\right)+\mathbf{Q}\left[B_{n}\right] \ln \left(\frac{\mathbf{Q}\left[B_{n}\right]}{\mathbf{P}\left[B_{n}\right]}\right)\right) \\
& \leq C \sum_{n=1}^{\infty}\left(\frac{2^{-n}}{n^{2}} 2^{n}+\frac{2^{-n}}{n} \ln \left(\frac{1}{n}\right)\right)<\infty
\end{aligned}
$$

(ii) and (iii) were shown above.

(iv) We have already observed that $\mathbf{Q}$ is the unique equivalent martingale measure for $S^{\text {large }}$ on $\mathcal{F}_{\infty}^{S}$ and that (70) holds true. Hence it follows from [S 01, Theorem 2.1] that $\left(\widehat{X}_{n}\right)_{n=0}^{\infty}:=\left(S_{n}^{1}\right)_{n=0}^{\infty}$ is the optimal investment process for the exponential utility function $U(x)=-e^{-x}$, and $\widehat{X}_{0}=a_{0}$. 
(v) For $S^{\text {small }}$ we have that $\mathbf{P} \in \mathcal{M}^{e}\left(S^{\text {small }}\right)$ and therefore it is the entropy-minimizing local martingale measure for $S^{\text {small }}$. This trivially implies that $\widehat{X}_{n} \equiv \widehat{X}_{0}$ is the optimal process starting at $\widehat{X}_{0}=a_{0}$.

Proof of Proposition 3 We define $S=\left(S_{n}\right)_{n=0}^{\infty}$ by choosing the following sequences $\left(a_{n}\right)_{n=0}^{\infty},\left(b_{n}\right)_{n=1}^{\infty},\left(q_{n}\right)_{n=1}^{\infty},\left(\widehat{q}_{n}\right)_{n=1}^{\infty}$ :

$$
a_{n}=-\left(2^{n}-1\right), \quad b_{n}=1, \quad q_{n}=2^{-n}, \quad \widehat{q}_{n}=1-e^{-2^{n}} .
$$

Here $\widehat{q}_{n}$ plays the role of $p_{n}$ in the above construction. The reason for this change of notation is, that we now shall also consider a probability measure $\mathbf{P}$ on $\mathcal{F}_{\infty}^{S}$ (under which $S$ is not a martingale) defined by $\frac{d \mathbf{P}}{d \widehat{\mathbf{Q}}}=c^{-1} e^{S_{\infty}}$, where the normalizing constant $c$ is chosen such that $c=\mathbf{E}_{\widehat{\mathbf{Q}}}\left[e^{S_{\infty}}\right]$. The expectation is well-defined, as $S_{\infty}$ is bounded from above. We have

$$
\begin{aligned}
& \mathbf{Q}\left[A_{n}\right]=2^{-n} \prod_{i=1}^{n-1}\left(1-q_{i}\right) q_{n} \approx 2^{-2 n} \quad \mathbf{Q}\left[B_{n}\right]=2^{-n} \prod_{i=1}^{n-1}\left(1-q_{i}\right) \approx 2^{-n} \\
& \widehat{\mathbf{Q}}\left[A_{n}\right]=2^{-n} \prod_{i=1}^{n-1}\left(1-\widehat{q}_{i}\right) \widehat{q}_{n} \approx 2^{-n} e^{-2^{n}} \quad \widehat{\mathbf{Q}}\left[B_{n}\right]=2^{-n} \prod_{i=1}^{n-1}\left(1-\widehat{q}_{i}\right) \approx 2^{-n} e^{-2^{n}} \\
& \mathbf{P}\left[A_{n}\right]=c^{-1} \cdot e^{-\left(2^{n}-1\right)} \widehat{\mathbf{Q}}\left[A_{n}\right] \approx 2^{-n} e^{-2^{n+1}} \quad \mathbf{P}\left[B_{n}\right]=c^{-1} e \widehat{\mathbf{Q}}\left[B_{n}\right] \quad \approx 2^{-n} e^{-2^{n}}
\end{aligned}
$$

For the entropy $H(\widehat{\mathbf{Q}} \mid \mathbf{P})$ we get, with $B=\bigcup_{n=1}^{\infty} B_{n}$ and $C>0$ sufficiently large,

$$
\begin{aligned}
H(\widehat{\mathbf{Q}} \mid \mathbf{P}) & =\sum_{n=1}^{\infty} \widehat{\mathbf{Q}}\left[A_{n}\right] \ln \left(\frac{\widehat{\mathbf{Q}}\left[A_{n}\right]}{\mathbf{P}\left[A_{n}\right]}\right)+\widehat{\mathbf{Q}}[B] \ln \left(\frac{\widehat{\mathbf{Q}}[B]}{\mathbf{P}[B]}\right) \\
& \leq C \sum_{n=1}^{\infty} 2^{-n} e^{-2^{n}} \ln \left(e^{2^{n}}\right)+\widehat{\mathbf{Q}}[B](\ln (c)-1)<\infty
\end{aligned}
$$

A similar calculation reveals that $H(\mathbf{Q} \mid \mathbf{P})=\infty$. More generally, for any local martingale measure $\widetilde{\mathbf{Q}} \in \mathcal{M}^{a}(S)$, such that $S$ fails to be uniformly integrable under $\widetilde{\mathbf{Q}}$, we have $H(\widetilde{\mathbf{Q}} \mid \mathbf{P})=\infty$. Indeed, the local $\widetilde{\mathbf{Q}}$-martingale $S$ is uniformly integrable iff $\lim _{n \rightarrow \infty}\left(2^{n}-1\right) \widetilde{\mathbf{Q}}\left[C_{n}\right]=0$ (compare Lemma 6 ). By the martingale property we have $\widetilde{\mathbf{Q}}\left[B_{n}\right]=\widetilde{\mathbf{Q}}\left[C_{n}\right]$. Hence, if $S$ fails to be uniformly integrable, we may find $\alpha>0$, and an infinite subset $I \subseteq \mathbb{N}$, such that $\widetilde{\mathbf{Q}}\left[B_{n}\right] \geq \alpha 2^{-n}$, for $n \in I$. This implies

$$
H(\widetilde{\mathbf{Q}} \mid \mathbf{P}) \geq \sum_{n \in I} \widetilde{\mathbf{Q}}\left[B_{n}\right] \ln \frac{\widetilde{\mathbf{Q}}\left[B_{n}\right]}{\mathbf{P}\left[B_{n}\right]}-e^{-1}=\infty .
$$

To show the optimality of $\widehat{\mathbf{Q}}$, fix $\widetilde{\mathbf{Q}} \in \mathcal{M}^{a}(S)$ with finite entropy $H(\widetilde{\mathbf{Q}} \mid \mathbf{P})$; we have seen that $S$ is uniformly $\widetilde{\mathbf{Q}}$-integrable. Applying the usual Fenchel inequality (see (13), (17), (18)) to $U(x)=-e^{-x}$ and $V(y)=y(\ln (y)-1)$, we have

$$
\begin{aligned}
& H(\widetilde{\mathbf{Q}} \mid P)=\mathbf{E}\left[V\left(\frac{d \widetilde{\mathbf{Q}}}{d \mathbf{P}}\right)\right]+1 \\
& \quad \geq \mathbf{E}\left[-e^{-S_{\infty}+\ln c}-\left(S_{\infty}-\ln c\right) \frac{d \widetilde{\mathbf{Q}}}{d \mathbf{P}}\right]+1=-c \mathbf{E}\left[e^{-S_{\infty}}\right]+\ln c+1
\end{aligned}
$$


where the equality holds iff $-V^{\prime}\left(\frac{d \widetilde{\mathbf{Q}}}{d \mathbf{P}}\right)=-\ln \left(\frac{d \widetilde{\mathbf{Q}}}{d \mathbf{P}}\right)=S_{\infty}-\ln (c)$ almost surely, i.e., iff $\widetilde{\mathbf{Q}}=\widehat{\mathbf{Q}}$. It follows from Theorem 1 that $S$ is the optimal process starting at $S_{0}=0$. The proof of Proposition 3 now is complete.

Proof of Proposition 4 Define $S=\left(S_{n}\right)_{n=0}^{\infty}$ by requiring that $\bar{S}:=2-S=(2-$ $\left.S_{n}\right)_{n=0}^{\infty}$ is the above example associated to the following parameters

$$
\bar{a}_{n}=-2^{n}+1, \quad \bar{b}_{n}=1, \quad q_{n}=2^{-n}, \quad \widehat{q}_{n}=\frac{1}{2} .
$$

Hence the process $S$ starts at $S_{0}=2$, takes its values in [1, $\infty$ [, and $S_{\infty}$ equals $a_{n}:=2^{n}+1$ and $b_{n}:=1$ on $A_{n}$ and $B_{n}$ respectively. The process $S$ is a uniformly integrable martingale with respect to $\widehat{\mathbf{Q}}$ but it is not uniformly integrable with respect to $\mathbf{Q}$. It is easy to see that

$$
\mathbf{Q}\left(A_{n}\right) \approx 2^{-2 n}, \quad \mathbf{Q}\left(B_{n}\right) \approx 2^{-n}, \quad \widehat{\mathbf{Q}}\left(A_{n}\right) \approx 2^{-2 n}, \quad \widehat{\mathbf{Q}}\left(B_{n}\right) \approx 2^{-2 n+1} .
$$

For $\mathbf{P}$ defined by $\frac{d \mathbf{P}}{d \widehat{\mathbf{Q}}}=\frac{S_{\infty}}{2}$ we obtain $\mathbf{P}\left(A_{n}\right) \approx 2^{-n}, \mathbf{P}\left(B_{n}\right) \approx 2^{-2 n}$.

Take a strictly concave, smooth utility function $U$ with $U(x)=\ln x$, for $x \geq 1$, and $U(x)=-e^{-x}$ for $x$ sufficiently small. With such a choice $V(y)=-\ln y-1$ when $y \in] 0,1]$ and $V(y)=y(\ln y-1)$ when $y$ is sufficiently large. Using the above asymptotics, we obtain that $\mathbf{E}\left[V \frac{d \mathbf{Q}}{d \mathbf{P}}\right]$ and $\mathbf{E}\left[V \frac{d \widehat{\mathbf{Q}}}{d \mathbf{P}}\right]$ are finite. As in Proposition 3, we verify that $\widehat{\mathbf{Q}}$ minimizes $\mathbf{E}\left[V \frac{d \widetilde{\mathbf{Q}}}{d \mathbf{P}}\right]$ over all absolutely continuous martingale measures $\widetilde{\mathbf{Q}}$, and that $S$ is the optimal process starting at $S_{0}=2$. The measure $\mathbf{Q}$ has finite $V$-expectation but, for $x_{0}=2$, the closed process $\left(\widehat{X}_{n}\left(x_{0}\right)\right)_{n \in \mathbb{N}_{0} \cup\{\infty\}}=\left(S_{n}\right)_{n \in \mathbb{N}_{0} \cup\{\infty\}}$, defined over the closed index set $\mathbb{N}_{0} \cup\{\infty\}$, fails to be a Q-submartingale.

The proof of proposition 4 now is complete.

Remark 2 The counter-examples constructed above are such that the processes $\left(S_{n}\right)_{n=0}^{\infty}$ are indexed by discrete time. It is not difficult to modify the construction such that these processes are indexed by continuous time $[0, T]$ and have continuous paths. It suffices to adapt the corresponding arguments from [S 93]. The details of this construction as well as an extensive discussion of the economic interpretation of Proposition 2 and other complementary results are carried out in the working version [S 02] of the present paper.

\section{A Appendix}

In the proof of Lemma 4 we have used the following result which is in the spirit of [DS 94, Lemma 4.5].

Proposition 5 Under the assumptions of Theorem 1, fix $x \in \mathbb{R}$ and a sequence $\left(H^{n}\right)_{n=1}^{\infty}$ of admissible integrands such that

$$
\lim _{n \rightarrow \infty} \mathbf{E}\left[U\left(x+\left(H^{n} \cdot S\right)_{T} \wedge(\widehat{H}(x) \cdot S)_{T}\right)\right]=u(x) .
$$


Then for any $[0, T]$-valued stopping time $\tau$

$$
\lim _{n \rightarrow \infty}\left(H^{n} \cdot S\right)_{\tau}=(\widehat{H}(x) \cdot S)_{\tau} \quad \text { in measure. }
$$

Proof Write $\widehat{H}$ for $\widehat{H}(x)$. Suppose there is $1>\alpha>0$ such that, for

$$
A_{n}=\left\{(\widehat{H} \cdot S)_{\tau} \leq\left(H^{n} \cdot S\right)_{\tau}-\alpha\right\}, \quad B_{n}=\left\{\left(H^{n} \cdot S\right)_{\tau} \leq(\widehat{H} \cdot S)_{\tau}-\alpha\right\}
$$

we have $\mathbf{P}\left[A_{n}\right] \geq \alpha$ or $\mathbf{P}\left[B_{n}\right] \geq \alpha$, for infinitely many $n$ 's. We assume that this holds true for $A_{n}$, the case of $B_{n}$ being similar.

Define $\tau^{n}=\tau \mathbf{1}_{A_{n}}+T \mathbf{1}_{\Omega \backslash A_{n}}$ and the concatenaded integrands

$$
\widetilde{H}^{n}=H^{n}+\left(\widehat{H}-H^{n}\right) \mathbf{1}_{\rrbracket \tau^{n}, \tau \rrbracket} .
$$

As in Lemma $4, \widetilde{H}^{n}$ is such that $\widetilde{H}^{n} \cdot S$ is a $\widehat{\mathbf{Q}}(y)$-supermartingale. By the optimality of $\widehat{H}$ (see the proof of Theorem 1)

$$
\mathbf{E}\left[U\left(x+\left(\widetilde{H}^{n} \cdot S\right)_{T}\right)\right] \leq \mathbf{E}\left[U\left(x+(\widehat{H} \cdot S)_{T}\right)\right] .
$$

On the other hand we may write the random variable $\left(\widetilde{H}^{n} \cdot S\right)_{T}$ as $f_{n}+g_{n}$ where $f_{n}=(\widehat{H} \cdot S)_{T} \mathbf{1}_{A_{n}}+\left(H^{n} \cdot S\right) \mathbf{1}_{\Omega \backslash A_{n}}$, and $g_{n}=\left(\left(H^{n} \cdot S\right)_{\tau}-(\widehat{H} \cdot S)_{\tau}\right) \mathbf{1}_{A_{n}}$ is a function supported by $A_{n}$ and greater than or equal to $\alpha$ on $A_{n}$.

The sequence $\left(f_{n}\right)_{n=1}^{\infty}$ tends in measure to $(\widehat{H} \cdot S)_{T}$ and satisfies

$$
\liminf _{n \rightarrow \infty} \mathbf{E}\left[U\left(x+f_{n}\right)\right] \geq \mathbf{E}\left[U\left(x+(\widehat{H} \cdot S)_{T}\right)\right] .
$$

It follows that

$$
\begin{aligned}
\sup _{n} \mathbf{E}\left[U\left(x+\left(\widetilde{H}^{n} \cdot S\right)_{T}\right)\right] \\
=\sup _{n} \mathbf{E}\left[U\left(x+f_{n}+g_{n}\right)\right]>\mathbf{E}\left[U\left(x+(\widehat{H} \cdot S)_{T}\right)\right] .
\end{aligned}
$$

Indeed, let $x_{0} \in \mathbb{R}$ be big enough such that $\mathbf{P}\left[x+(\widehat{H} \cdot S)_{T} \geq x_{0}\right]<\frac{\alpha}{2}$ and let $\beta:=U^{\prime}\left(x_{0}+2 \alpha\right)$. Then

$$
\begin{aligned}
& \sup _{n} \mathbf{E}\left[U\left(x+f_{n}+g_{n}\right)\right] \\
& \geq \sup _{n} \mathbf{E}\left[U\left(x+f_{n}\right)+\alpha U^{\prime}\left(x+f_{n}+\alpha\right) \mathbf{1}_{A_{n}}\right] \\
& \geq \sup _{n}\left\{\mathbf{E}\left[U\left(x+f_{n}\right)\right]+\alpha \mathbf{P}\left[A_{n} \cap\left\{x+f_{n}<x_{0}+\alpha\right\}\right] \beta\right\} \\
& \geq \mathbf{E}\left[U\left(x+(\widehat{H} \cdot S)_{T}\right)\right]+\frac{\alpha^{2} \beta}{2} .
\end{aligned}
$$

This proves (103) which yields the desired contradiction. 


\section{References}

[AS 94] J.P. Ansel, C. Stricker, (1994), Couverture des actifs contingents et prix maximum. Ann. Inst. Henri Poincaré, Vol. 30, pp. 303-315.

[BF 02] F. Bellini, M. Fritelli, (2002), On the existence of minimax martingale measures. Mathematical Finance, Vol. 12, No. 1, pp. 1-21.

[C 75] I. Csiszar, (1975), I-Divergence, Geometry of Probability Distributions, and Minimization Problems. Annals of Prob.

[DGRSSS 02] F. Delbaen, P. Grandits, T. Rheinländer, D. Samperi, M. Schweizer, C. Stricker, (2002), Exponential hedging and entropic penalties. Mathematical Finance, Vol. 12, No. 2, pp. 99-123.

[DS 94] F. Delbaen, W. Schachermayer, (1994), A General Version of the Fundamental Theorem of Asset Pricing. Math. Annalen, Vol. 300, pp. 463-520.

[DS 97] F. Delbaen, W. Schachermayer, (1997), The Banach Space of Workable Contingent Claims in Arbitrage Theory. Annales de l' I.H.P., Vol. 33, No. 1, pp. 113-144.

[DS 98a] F. Delbaen, W. Schachermayer, (1998), A Simple Counter-Example to Several Problems in the Theory of Asset Pricing, which arises in many incomplete markets. Mathematical Finance, Vol. 8, pp. 1-12.

[DS 98b] F. Delbaen, W. Schachermayer, (1998), The Fundamental Theorem of Asset Pricing for Unbounded Stochastic Processes. Mathematische Annalen, Vol. 312, pp. 215-250.

[E 81] N. El Karoui, (1981), Les aspects probabilites du controle stochastique. Ecole d'Eté de Probabilités de Saint-Flour IX-1979 (P.L. Hennequin, editor), Springer Lecture Notes in Mathematics, Vol. 876, pp. 74-238.

[E 80] M. Emery, (1980), Compensation de processus à variation finie non localement intégrables. Séminaire de Probabilités XIV, Springer Lecture Notes in Mathematics, Vol. 784, pp. 152-160.

[F 00] M. Fritelli, (2000), The minimal entropy martingale measure and the valuation problem in incomplete markets. Mathematical Finance, Vol. 10, pp. 39-52.

[GR 02] P. Grandits, T. Rheinländer, (2002), On the minimal Entropy Martingale Measure. Annals of Probability, Vol. 30, No. 3, pp. 1003-1038.

[HP 81] J.M. Harrison, S.R. Pliska, (1981), Martingales and Stochastic integrals in the theory of continuous trading. Stoch. Proc. \& Appl., Vol. 11, pp. 215-260.

[J79] J. Jacod, (1979), Calcul stochastique et problèmes de martingales. Lecture Notes in Mathematics, Vol. 714. 
[Ka 02] Yu.M. Kabanov (2002), personal communication.

[K 01] J. Kallsen, (2001), Utility-Based Derivative Pricing in Incomplete Markets. Mathematical Finance: Bachelier Congress 2000 (H. Geman, D. Madan, St.R. Pliska, T. Vorst, editors), pp. 313-338, Springer.

[KS 02a] Yu.M. Kabanov, C. Stricker, (2002), On the optimal portfolio for the exponential utility maximization: remarks to the six-author paper. Mathematical Finance, Vol. 12, No. 2, pp. 125-134.

[KS 02b] Yu.M. Kabanov, Ch. Stricker, (2002), On the true submartingale property d'après Schachermayer. Séminaire de Probabilités XXXVI, Springer Lecture Notes in Mathematics.

[KLSX 91] I. Karatzas, J.P. Lehoczky, S.E. Shreve, G.L. Xu, (1991), Martingale and duality methods for utility maximisation in an incomplete market. SIAM Journal of Control and Optimisation, Vol. 29, pp. 702-730.

[KrS 99] D. Kramkov, W. Schachermayer, (1999), The Asymptotic Elasticity of Utility Functions and Optimal Investment in Incomplete Markets. Annals of Applied Probability, Vol. 9, No. 3, pp. 904-950.

[P 90] P. Protter, (1990), Stochastic Integration and Differential Equations. Applications of Mathematics, Vol. 21.

[RW 87] L.C.G. Rogers, D. Williams, (1987), Diffusions, Markov Processes and Martingales, Volume 2: Itô Calculus. Wiley, Chichester New York.

[S 93] W. Schachermayer, (1993), A Counter-Example to several Problems in the Theory of Asset Pricing. Math. Finance, Vol. 3, pp. 217-229.

[S 01] W. Schachermayer, (2001), Optimal Investment in Incomplete Markets when Wealth may Become Negative. Annals of Applied Probability, Vol. 11, No. 3, pp. 694-734.

[S 02] W. Schachermayer, (2002), How Potential Investments may Change the Optimal Portfolio for the Exponential Utility. Working Paper, (28 pages), Vienna University of Technology.

[St 02] E. Strasser, (2002), Necessary and sufficient conditions for the supermartingale property of a stochastic integral with respect to a local martingale. Preprint, to appear in Séminaire de Probabilités XXXVII.

[Str01] Ch. Stricker, (2001), Simple strategies in exponential utility maximization. Preprint of the Université de Franche-Comté. 